\title{
Aspects and Challenges of Unmanned Aircraft Systems Safety Assurance and Certification for Advanced Operations.
}

\author{
Michail K. Karyotakis ${ }^{1}$ \\ Cranfield University PhD Student, Patra, Peloponnese, 26334, Greece \\ D. Panagiotakopoulos ${ }^{2}$, G. Braithwaite ${ }^{3}$, A. Tsourdos ${ }^{4}$ \\ Cranfield University, Bedfordshire, MK43 OAL, United Kingdom
}

Like manned aviation, a Safety Management System (SMS) needs to be developed for Unmanned Aircraft Systems (UAS) taking into account their unique characteristics, and the huge variety of different operations they can perform. Towards developing a SMS for unmanned aviation this paper focuses on Safety Assurance and Certification for advanced UAS operations. Based on the manned aviation practices and the Concepts of Operations (ConOps) that have been developed for UAS, this paper examines two indicative operational scenarios (OS) in unmanned aviation identifying the gaps in the view of the safety assurance and certification processes. The findings form the basis for the proposal and development of a new safety management framework for certain UAS operations. The examination of OS shows that operation-centric operational approvals as well as faster integration of UAS to the current airspace may be possible under certain conditions.

\section{Nomenclature}

$\begin{array}{ll}\text { AGL } & =\text { Above Ground Level } \\ \text { aircraft } & =\text { refers to manned and unmanned aircraft } \\ \text { C3 } & =\text { Command, Control, and Communication } \\ f t & =\text { feet } \\ \text { helicopter } & =\text { refers to manned helicopter } \\ \text { MSL } & =\text { Mean Sea Level (height measure from sea level) } \\ \text { safety } & =\text { aviation safety } \\ \text { UAS } & =\text { refers to any kind of unmanned aircraft }\end{array}$

\section{Introduction}

Today, some state-of-the-art UAS are being used for several reasons, serving humans in many different applications. UAS are being used mainly for purposes that manned aircraft used to serve. UAS operations take place in parts of the airspace that are less used by manned aircraft like the airspace below $500 \mathrm{ft}$ above ground level (AGL). However, in the future UAS are expected to perform and materialize innovative ideas like urban air-taxis and providing first aid services in urban and remote areas just to name some. Inevitably, this will force UAS to fly in almost any part of the airspace both controlled and uncontrolled. UAS will gradually operate in the entire spectrum of the airspace and interact with the Air Traffic Control (ATC) and other traffic as well. Today, UAS are being used not

\footnotetext{
${ }^{1}$ Rank: Major, F-16 Fighter Pilot and Instructor, Hellenic Fighter Weapons School, Hellenic Air Force; PhD Student at School of Aerospace, Transport and Manufacturing (SATM).

${ }^{2}$ Head of UTM Research, School of Aerospace, Transport and Manufacturing (SATM).

${ }^{3}$ Director of School of Transport at SATM.

${ }^{4}$ Head of Centre for Autonomous and Cyber-Physical Systems and Director of Research at SATM.
} 
only for military and civil protection purposes but also for recreational and private activities. Like in manned aviation, a Safety Management System (SMS) needs to be developed for UAS, hereinafter referred as SMS(U), taking into account their unique characteristics and capabilities.

The SMS for manned aviation consists of four components: 1. Safety Policy \& Objectives, 2. Safety Risk Management, 3. Safety Assurance, and 4. Safety Promotion. (Ref. [19]). The European Union Aviation Safety Agency (EASA), defines three categories of UAS operations: 1. 'Open', 2. 'Specific', and 3. 'Certified'. This is an operationdriven categorization. In other countries like the United States of America (USA) and Australia similar categorizations are made based on the UAS characteristics and performance. The huge variety of UAS cannot be covered by a single developing approach for a SMS(U). For example, the Northrop Grumman Global Hawk has a maximum ferry range of 12,300 nautical miles and a maximum altitude of $60,000 \mathrm{ft}$. It has a maximum endurance of more than 32 hours and its maximum speed is 310 knots. On the other edge of the capabilities spectrum there are numerous UAS like the DJI Mavic 2 Pro that are available to the general public and can climb to 2,000 ft or higher and accelerate to approximately 70 kilometers per hour. (Ref. [6]). So, the framework for a SMS(U) needs to be performance-based and operationoriented.

This paper focuses on developing a framework for the 'Safety Assurance' component focusing on advanced UAS operations. Certification is considered as part of Safety Assurance. Two OS are examined in the context of unmanned aviation and results are extracted concerning the safety risk management. In Part VII, the Manned Aviation Legacy, in the domains of safety assurance and certification and also how these two aspects affect the management of safety risks, are outlined. Next, the Unmanned Aviation Perspective is described in Part VIII. Based on the existing literature on safety assurance and certification, Part IX provides the OS through which the Recommendations and Findings are provided in Part X.

\section{Background}

One critical aspect of manned and unmanned aviation is the airspace where operations using UAS or conventional aircraft take place. Today, the Air Traffic Management (ATM) infrastructure has been developed according to manned aviation specifics and safety standards. There is an ongoing process towards transforming the existing ATM capabilities into a new age of ATM that will be able to handle both manned and unmanned air traffic and at the same time preserving safety and efficiency. For unmanned aviation the concept of ATM is transformed to Unmanned Traffic Management (UTM). UTM services, concepts, infrastructure, and procedures are expected to facilitate the handling of UAS traffic and finally the integration of UAS to current airspace.

The main concept of UTM is the U-Space, as described in the Single European Sky ATM Research (SESAR) Concept of Operations for European UTM Systems (CORUS) Concept of Operations Vol.1 (2019) (Ref. [35]). CORUS introduced the term Very Low Level (VLL) airspace as the airspace below the minimum safe altitudes that manned aircraft must remain above which are $500 \mathrm{ft}$ and $1,000 \mathrm{ft}$ depending on the phase of flight and the area of operation, respectively. (Ref. [36]). In addition, the airspace below 1,000 ft over urban areas is expected to be used for a new kind of operation, which is the Urban Air Mobility (UAM).

According to Ref. [31] it has been found that 'the Airport Shuttle and Air Taxi markets are viable, with a significant total available market value in the U.S. of $\$ 500$ billion, for a fully unconstrained ${ }^{5}$ scenario.' In the UK, the world-first electric Urban Airport is under construction and estimated to be ready not later than November 2021. (Ref. [5]). UAM has drawn the attention of several companies like Airbus, Boeing, Vertical Aerospace Ltd, and E-Hang to name a few, with some expected to make their first commercial flight by 2025. In 2019, Airbus tested for first time the CityAirbus which is an $\mathrm{eVTOL}^{6}$ remotely piloted aircraft capable of carrying four passengers. (Ref. [3]). In some cases, UAM is expected to be performed initially with at least one pilot onboard but it is envisaged to become part of the unmanned era of aviation soon. An indicative example is the Y6S Plus, a multi-role eVTOL aircraft which in the beginning will be operated by a single pilot. (Ref. [34]). Regarding UAS, amongst the huge variety of their different applications an indicative example is the Ambular 3.0 eVTOL emergency response aircraft which is expected to be fully autonomous or remotely piloted. (Ref. [33]).

Based on ICAO, the Safety Assurance is mainly based on performance monitoring. The term performance refers to the staff, the safety systems that are in place, the procedures, and the safe performance of the system as a whole. A system could be a specific operation, an operator, or a critical stakeholder. What should be noted in UAS operations is the number of potential stakeholders (usually bigger than manned aviation) for one single operation. As the number

\footnotetext{
5 'Unconstrained' - meaning that people could fly using UAM vehicles anytime to go anywhere without any constraint.

${ }^{6}$ eVTOL - electric vertical takeoff and landing. A technology that is widely used in UAM and in many UAS.
} 
of the potential stakeholders increases the probability of system performance shortfalls also increases rendering the overall safety performance questionable. In addition, setting Safety Performance Indicators (SPIs) and Safety Performance Targets (SPTs) may differ in unmanned aviation. Compared to the SPTs and SPIs analyzed in the SMS, the standards of setting these metrics need to be stricter in the SMS(U). For example, in manned aviation a SPT and a SPI could be 'zero mid-air collisions (MACs) during a specified time period' and 'number of flight rules violations by aircrew during the same time period', respectively. In unmanned operations the aforementioned SPT could be the same and for the same example. However, the SPI should be different, meaning that in UAS operations the number of flight rules violations needs to be zero in order to achieve zero mid-air collisions with manned aircraft. This couple of SPT and SPI is also related to different mitigation strategies, crew (UAS pilots and operators) certification, standards, and procedures.

Regarding Certification, there is a twofold issue. One side is the fact that the sudden advent of UAS operations did not allow the adequate preparation of potential RIC with the appropriate piloting skills and aviation safety culture. The other side is linked to the great variety of current UAS that are expected to perform and, in some cases, replace the respective manned aircraft operations, incorporating different levels of technology and automation. Besides the required aeronautical knowledge based on Ref. [25], certification should take into account the fact that Visual Line of Sight (VLOS) and Beyond Visual Line of Sight (BVLOS) UAS flights correspond to some unique differences of how they might be executed and handled from the ATM and UTM services. In regard to avoiding collisions, not every UAS is equipped with proximity warning systems like Traffic Collision Avoidance Systems (TCAS) and Ground Proximity Warning Systems (GPWS).

\section{Scope}

This paper deals with UAS not only as the flying component of unmanned operations but also with the Remote Pilot in Command (RIC) and the ATC services. It also, deals with operations that are conducted under the 'Specific' category regulations. The size and complexity of these UAS as well as the complexity of the respective operations drive the necessity to monitor and mitigate any possible safety risk, especially the risk of MAC with other manned aircraft (and unmanned) and the risk of hitting people on the ground or destroying properties, ground collision (GC).

The UAS operations that are contained in the scope of this paper are related to people transportation, and transferring of goods in urban and rural areas. These types of operations are considered as 'Advanced' and may have the following common characteristics:

- $\quad$ They will need to be conducted mostly in BVLOS conditions;

- $\quad$ They will be conducted in every flight level and not only in VLL airspace;

- They will need to interact with other manned and unmanned traffic in a great extent due to their operational range;

- They will need to interact with the air traffic services when this interaction would be deemed to be necessary; and

- They could pose significant danger to people on ground and on other air traffic if the respective flight parameters might deteriorate.

Bearing in mind that a SMS(U) should be oriented towards minimizing the safety risks through effective management and providing solutions in order to simplify and facilitate the operation approval process, it could be derived that such a SMS(U) covers UAS operations that may pose greater safety risks than others. According to EASA, a UAS operator of the 'Open' category is not required to perform a safety risk assessment in order to take approval for operation. Moreover, reasons like the huge variety, the increasing rate of utilization of UAS applicable to operate in the 'Open' category, the potential stakeholders, the affected airspace, and the safety risks (in terms of frequency and severity) necessitate studying of the 'Open' category separately. So, 'Open' category operations are out of the scope of this paper. In addition, operations of the 'Certified' category are mainly conducted with UAS that have identical capabilities, airworthiness standards and safety provisions to manned aircraft, and thus are also considered to be out of the scope of this paper. Usually, such UAS are designed according to the Certification Standards (CS) used for manned aircraft. For example, the Global Hawk is designed based on the CS- 25 which corresponds to a fiftyseat airliner. (Ref. [11]).

Finally, international UAS flights are also out of the scope of this paper because they require the collaboration between multiple air traffic services from different countries which may not have the required infrastructure to accommodate them as a result of a respective technological gap between these countries. Furthermore, till the time of writing no international UAS flights are being conducted and as a result there are no data that can acquired. Table 1 shows the operations that are in the scope of this paper. 
Table 1 Operations in the Scope of this paper.

\begin{tabular}{|c|c|c|c|c|}
\hline \multirow{2}{*}{\multicolumn{2}{|c|}{$\begin{array}{l}\text { Note: } \\
\text { All flights performed in } \mathrm{VMC}^{7} \text {. }\end{array}$}} & \multicolumn{3}{|c|}{ Operating Airspace } \\
\hline & & Controlled & Uncontrolled & Urban \\
\hline \multirow{2}{*}{$\begin{array}{c}\text { UAS } \\
\text { Category } \\
\& \\
\text { LOS } \\
\text { condition }\end{array}$} & 'Specific’ BVLOS & Yes & Yes & $\begin{array}{l}\text { Yes, but only in VLL } \\
\text { airspace. }\end{array}$ \\
\hline & $\begin{array}{l}\text { 'Specific' with certified } \\
\text { equipment on board. }\end{array}$ & Yes, only for BVLOS & Yes, only for BVLOS & Yes, only for BVLOS \\
\hline
\end{tabular}

\section{Objectives}

\section{A. Main Objective}

The main objective is to shed light on the aspects and challenges of UAS safety assurance and certification for advanced operations in order to provide the basis for the creation of the respective component of a SMS(U).

\section{B. Sub Objectives}

The paper aims at providing qualitative results by achieving the following four sub objectives:

1) Highlighting the most critical aspects of safety assurance and certification;

2) Compare the safety assurance and certification in manned and unmanned aviation;

3) Analyze two operations performed with manned and unmanned aircraft and revealing the gaps that have to be bridged regarding to the safety assurance and certification aspects of UAS operations.

\section{Related Work}

Despite the fact that the integration provided today is limited and under certain assumptions and conditions, ConOps have been formulated under the basic assumption of preserving safety through safety risk management and providing the procedures and the infrastructure that are needed for the execution of certain UAS operations under certain conditions.

\section{A. CORUS (EU)}

In Europe, the CORUS project has been developed by JARUS ${ }^{8}$ in collaboration with SESAR. CORUS was introduced in 2019, covering the regulations and the services that should be in place for VLL UAS operations. (Ref. [35]). It takes into consideration the existence, development where needed and the involvement of certain U-space stakeholders that are depicted in Fig. 1. (Ref. [36]). CORUS suggests four phases of U-space implementation starting from U1 to U4. This progression is linearly linked to the involvement of U-space stakeholders and the services they may provide and the technological advancements in the field of UAS manufacturing and digital information and data exchanging. U4 corresponds to the full integration phase of UAS.

As the U-space advances towards U4, the integration of UAS expands across the airspace and finally U4 corresponds to the full integration of UAS in the current airspace. In order to cover this giant leap from segregation of UAS operations to fully integrating them in the airspace, CORUS suggests three types of VLL airspace: $X, Y$ and $Z$. Actually, these types of airspace correspond to different levels of U-space evolvement because they are linked to certain provisions of services required for different kind and complexity of unmanned flights. Table 2 shows the access requirements for the three types of airspace. (Ref. [35]). Table 2 shows that moving from $\mathrm{X}$ to $\mathrm{Z}$ type of airspace the UAS operations become riskier and more complex. Therefore, more mitigating measures and services need to be in place for the safe operation of manned and unmanned aircraft. The relation between the three types of airspace and the U-space phases leads, according to CORUS, to the coexistence and thus the integration of UAS operations to the current airspace through the integration of the $\mathrm{X}, \mathrm{Y}$ and $\mathrm{Z}$ types to the airspace classes as known today.

For all of the three airspace types some services are mandated and some others that are optional in $\mathrm{X}$ airspace become mandated in the $\mathrm{Z}$ type. Moreover, some services exist only in the corresponding U-space phase. The reason is that the required technology required for some services has not been developed yet. Such technological

\footnotetext{
${ }^{7}$ VMC - Visual Meteorological Conditions. The opposite of Instrument Meteorological Conditions (IMC). Usually, VMC and IMC corresponds to Visual Flight Rules (VFR) and Instrument Flight Rules (IFR) flights, respectively.

${ }^{8}$ JARUS - Joint Authorities for Rulemaking on Unmanned Systems.
} 
improvements could be linked to the U-space services evolution and/or the UAS themselves. Indeed, in Ref. [35], it is noted that type $\mathrm{Y}$ airspace is available from $\mathrm{U} 2$ onwards and type $\mathrm{Z}$ airspace is available from U3 onwards.

The setting of all the aforementioned parameters meaning the UAS stakeholders, service providers, types of airspace, different services provided, according to the type of operation and the airspace affected, leads to the configuration of the VLL airspace in a way that conflicts between manned and unmanned aircraft could be avoided and in parallel UAS could perform safe enough in order to minimize the risks for properties on ground. The services that are already available as well as the services that are envisaged are listed in Table 3, based on Ref. [35].

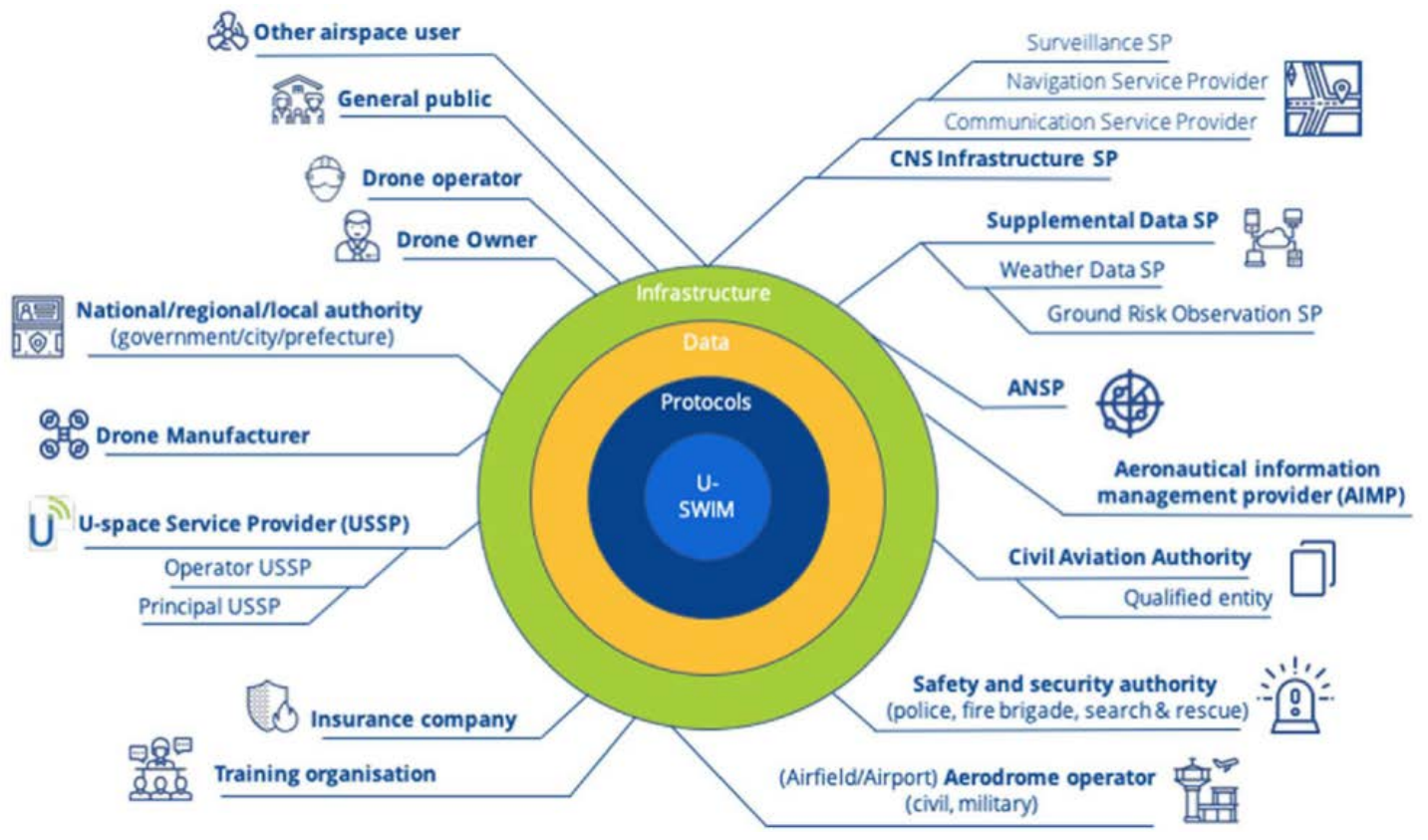

Fig. 1 U-space stakeholders.

Table 2 Types of airspace and their requirements for access.

\begin{tabular}{|c|c|}
\hline Type & Access requirements \\
\hline $\mathrm{X}$ & $\begin{array}{l}\text { - There are few basic requirements on the operator, the pilot or the drone. } \\
\text { - } \quad \text { The pilot remains responsible for collision avoidance. } \\
\text { - } \quad \text { VLOS and EVLOS flight are easily possible. } \\
\text { - Other flight modes in X require (significant) risk mitigation. }\end{array}$ \\
\hline $\mathrm{Y}$ & $\begin{array}{l}\text { - } \quad \text { An approved operation plan } \\
\text { - } \quad \text { A pilot trained for Y operation } \\
\text { - A remote piloting station connected to U-space } \\
\text { - A drone and remote piloting station capable of position reporting when available } \\
\text { Y airspaces may also have specific technical requirements attached to them }\end{array}$ \\
\hline $\mathrm{Z}$ & $\begin{array}{l}\text { - } \quad \text { An approved operation plan } \\
\text { - } \quad \text { A pilot trained for } Z \text { operation and/or a compatible, connected automatic drone } \\
\text { - A remote piloting station connected to U-space } \\
\text { - } \quad \text { A drone and remote piloting station capable of position reporting } \\
\text { drone be fitted with collaborative detect and avoid system for collision avoidance. }\end{array}$ \\
\hline
\end{tabular}




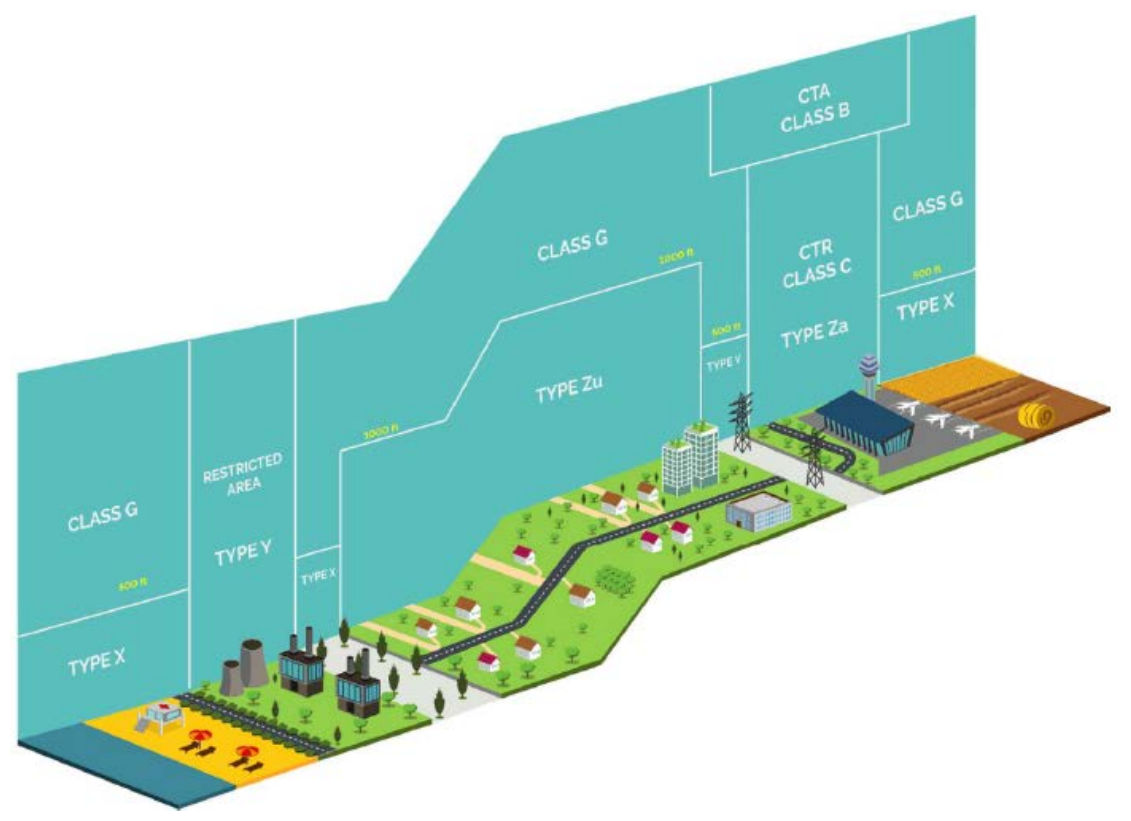

Fig. $2 \mathrm{X}, \mathrm{Y}$, and $\mathrm{Z}$ volumes.

Table 3 Services available per Airspace type per U-space Phase.

\begin{tabular}{lccc}
\hline Service & $\mathbf{X}$ & $\mathbf{Y}$ & $\mathrm{Z}$ \\
\hline Registration & Mandated & Mandated & Mandated \\
\hline e-identification & Mandated & Mandated & Mandated \\
\hline Geo-awareness & Mandated & Mandated & Mandated \\
\hline Drone Aeronautical Information Publication & Mandated & Mandated & Mandated \\
\hline Geo-fencing provision & Mandated & Mandated* & Mandated \\
\hline Incident / accident reporting & Mandated & Mandated & Mandated \\
\hline Weather information & Mandated & Mandated & Mandated \\
\hline Position report submission sub-service & Recommended & Mandated* & Mandated \\
\hline Tracking & Optional & Mandated* & Mandated \\
\hline Drone operation plan processing & Optional & Mandated & Mandated \\
\hline Emergency management & Optional* & Mandated* & Mandated \\
\hline Monitoring & Optional & Mandated* & Mandated \\
\hline Procedural interface with ATC & Optional+ & Mandated + & Mandated \\
\hline Strategic conflict resolution & No & Mandated & Mandated \\
\hline Legal recording & Optional+ & Mandated* & Mandated \\
\hline Digital logbook & Optional+ & Mandated* & Mandated \\
\hline Traffic information & Optional & Mandated & Offered \\
\hline Geospatial information service & Optional & Optional & Mandated* \\
\hline Population density map & Optional & Optional & Mandated* \\
\hline Electromagnetic interference information & Optional & Optional & Mandated* \\
\hline Navigation coverage information & Optional & Optional & Mandated* \\
\hline Communication coverage information & Optional & Optional & Mandated* \\
\hline Collaborative interface with ATC & Optional+ & Mandated+ & Mandated \\
\hline Dynamic capacity management & No & Mandated* & Mandated \\
\hline Tactical conflict resolution & No & No & Mandated \\
\hline U-space Phase & U1 & U2 & U3 \\
\hline & & + when needed & $*$ where available
\end{tabular}


Regarding to conflict management, CORUS suggests three layers being the following: Strategical (pre-tactical) de-confliction, Tactical separation provision and Collision avoidance using Detect and Avoid (DAA) systems. These layers are derived from practices already used in manned aviation. Indeed, all of the three are used in manned aviation for aircraft deconfliction. The Strategical layer is shaped by the flight plans that are filed, the Tactical layer is mainly an ATC function, and the Collision avoidance has the form of See and Avoid (SAA) (pilot on board) and is achieved through cooperative systems for separation (e.g., TCAS). The difference in unmanned aviation is that the ATC function is not scalable for every UAS, which would need higher levels of automation in order to receive traffic information timely and accurately. Furthermore, the lack of cooperative separation systems that can be compensated by the SAA in manned aircraft, has to be compensated by DAA systems in unmanned aircraft. DAA systems performance is subject to the available technology, current weather, and atmospheric conditions.

According to Ref. [36], the Strategical layer takes place before the flight and during the planning of the UAS operation. Through this planning UAS are expected to avoid any airborne conflicts by choosing to operate on different routes and avoid entering specific areas. If what is planned cannot be followed exactly then the Tactical layer takes action. A major difference to the previous layer is that at this stage UAS are airborne and either operating on a VLOS or BVLOS environment. The core part of the Tactical layer is the assurance of the biggest possible situational awareness (SA) both on UAS pilots and on the traffic services that may need to be aware of certain UAS operations. The main objective of the Tactical provisions is to provide information to UAS operators and they in turn execute the required changes of flight parameters (e.g., speed, altitude, heading, etc.) providing that the relevant information has been received and thus avoiding possible MAC. If both the aforementioned layers fail then the collision avoidance rests to the airborne platforms by the use of DAA systems. Based on Ref. [36], X airspace type offers no separation services and all the responsibility for safe operation is with the remote pilot, $\mathrm{Y}$ airspace type provides strategic conflict resolution and the $\mathrm{Z}$ airspace type provides strategic and tactical conflict resolution.

As far as emergency situations are concerned, in Ref. [36], it is stated that 'an emergency for a drone/operator is an incident/accident which causes the drone to be out of control.' From the point of view of the available services to UAS under an emergency situation, the U-space incorporates an Emergency Management Service (EMS). According to Ref. [36], EMS is intended to provide assistance to RIC and at the same time communicate any information related to the emergency to those who may be interested (e.g., manned aviation, other drone pilots and air traffic services).

Finally, it should be noted that a UTM system may provide U-space services such as Emergency Management, only after the development of key technologies and if certain steps can be fulfilled (i.e., UAS remote identification (RID), tracking, monitoring and data exchange) in real or near-real time.

\section{B. FAA UTM ConOps (USA)}

In the USA, in 2020, the Federal Aviation Administration (FAA) published the UTM ConOps V2.0. Ref. [15]. In the context of this paper, the basic aspects of this ConOps are the Performance Authorization, Airspace Authorization, BVLOS operations in controlled airspace, Remote Identification, UAS Volume Reservations and Data archiving and access. According to Ref. [15], this ConOps 'focuses on UTM operations below $400 \mathrm{ft}$ AGL, and addresses increasingly complex UTM operations within and across both uncontrolled (Class G) and controlled (Classes B, C, D, E[surface]) airspace environments.' Figure 3 shows the relation between airspace and services provided, as shown in Ref. [15]. In order to make these services available to every UAS operator and to manned aircraft if needed, the UTM ConOps is based on information sharing between operators. This information, according to Ref. [15], may include flight intent and airspace constraints.

Regarding the UTM system participants, in the context of this paper the more critical participants or UAS stakeholders are considered to be the Operator, the Remote Pilot in Command, the UAS Service Suppliers (USS), UAS Supplemental Data Service Providers (UAS SDSPs) and the USS Network. Based on Ref. [15], USS is the means of helping UAS operators meet operational requirements. USS provisions cover operations planning, intent sharing, strategic and tactical deconfliction, conformance monitoring, Remote ID, Airspace Authorization, airspace management functions and management of off-nominal situations. (Ref. [15]). The UAS SDSPs provide additional data to perform a flight such as terrain and obstacle data, specialized weather data, surveillance and constraint information. Specifically, the following five elements, as listed in Ref. [15], ensure the safe execution and management of unmanned flights:

1) Issuance of Performance Authorizations that ensure Operator performance requirements are met,

2) Airspace Authorizations - required for operations in controlled airspace,

3) Operation Planning that supports the sharing of flight intent

4) Airspace constraint and advisory information dissemination, and

5) Use of services, technologies, and equipage to de-conflict operations. 


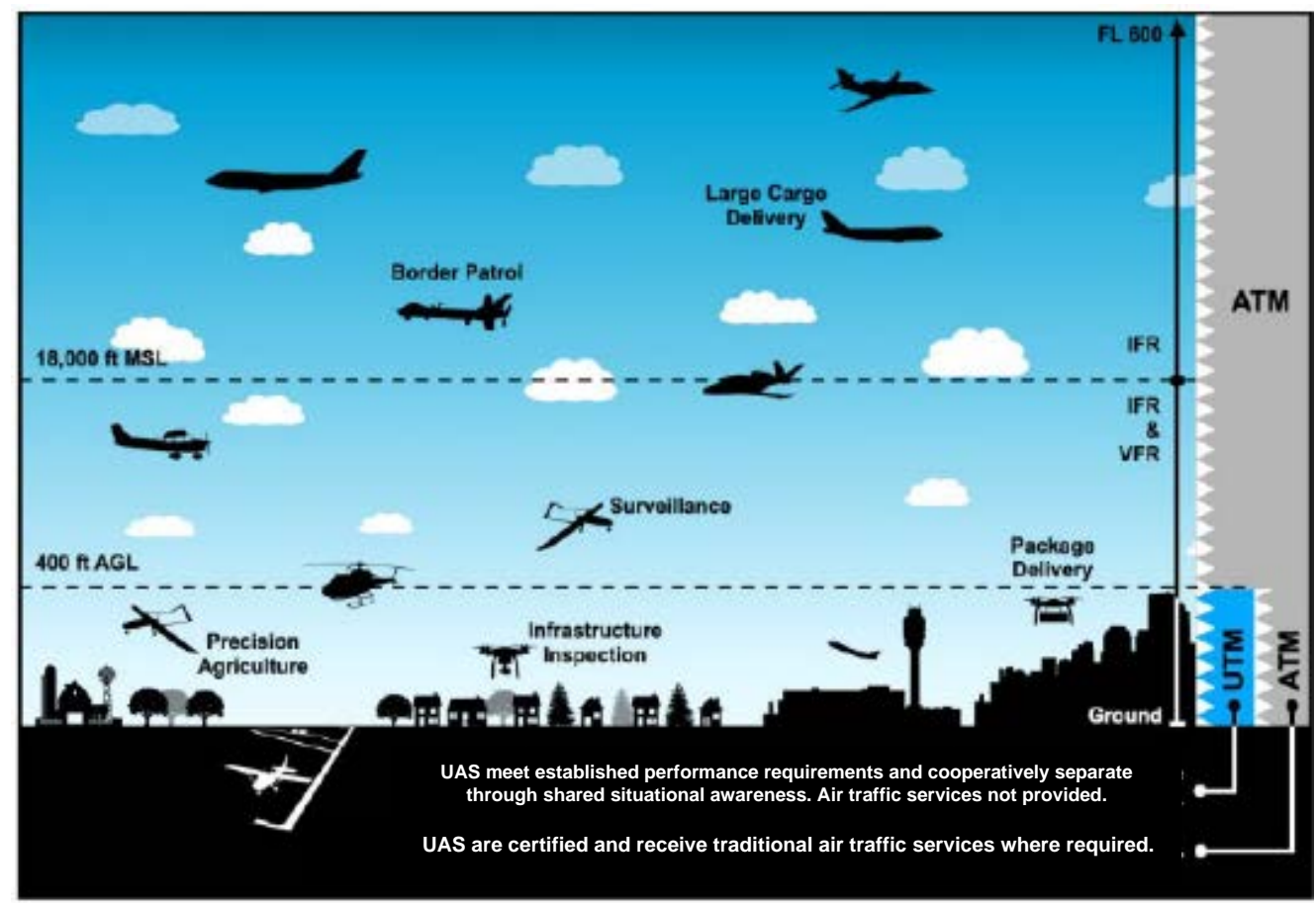

Fig. 3 Operational context of UTM services.

Performance authorizations are basically required for operations in uncontrolled airspace. The performance requirements may not be the same for every operation and in every part of the uncontrolled airspace. So, different performance requirements may apply in order to take into account the risk of an operation, the vicinity to a habituated area, the complexity of an operation and other operating factors that determine the safety risk of an operation.

In the case of controlled airspace operations, an airspace authorization is required apart from the respective performance authorization. Moreover, the intention of operation makes known what type of operation will take place and describes the exact location or for example the exact route that a UAS will follow. These data are used by the USS Network and the Service providers to disseminate information related to an unmanned flight that may be in progress and also alerts the UAS operators and/or RICs for any part of the airspace that UAS must not violate. In Ref. [15], it is stated that 'UAS Volume Reservations (UVRs) may be established when activities on the ground, or in the air, present a potential risk to UTM safety interests. [...]. UVRs are generally short in duration, have specified airspace boundaries, and have an established start and end times.'

Regarding manned aircraft interaction with the UTM system, manned aircraft could participate in the UTM system either passively or actively and this participation is encouraged but not mandated. Ref. [15]. Passive participation means that manned aircraft may use information provided by the USS Network in order to increase their SA but they do not share their flight intent with UAS operators. One the other hand, Active participation means that manned aircraft share their flight intent with UAS operators and they can provide additional data regarding their operations.

As far as the management of safety risks are concerned, UAS operators are ultimately responsible for avoiding any conflict between different unmanned aircraft as well as between unmanned and manned aircraft. They are also responsible for avoiding weather, terrain and other hazards that may impose additional safety risks to their operations. The deconfliction layers are basically the same to those mentioned in the CORUS document: strategic and tactical deconfliction and collision avoidance (MACs and GCs) based on the available technology. In particular, regarding the deconfliction/separation services provided in each type of airspace, in Ref. [15] it is described that in uncontrolled airspace (Class G) no deconfliction/separation services are provided and the operators, manned and unmanned are ultimately responsible for achieving the required separation. Conversely, in controlled airspace, either below or above $400 \mathrm{ft}$ AGL, deconfliction/separation services and traffic information are provided based on the complexity and the type of each operation.

A critical part of unmanned operations is the RID which is also mentioned in the CORUS document. For UAS operations and especially for BVLOS cases RID is the means of checking the level of conformity with flight rules and advisories issued by the ATC. This is achieved by the RID message that each UAS may transmit. According to Ref. 
[15], the RID message may contain but not limited to, the unique identification information of a UAS and its location. RID information can be transmitted using the proposed methods of Direct Broadcast and Network Publishing as proposed by the RID Aviation Rulemaking Committee Report 2017 cited by the UTM ConOps V2.0. The difference between the two methods is that in Direct Broadcast data are transmitted with no particular recipient and can be received by everyone in the transmission range. On the other hand, in Network Publishing data are published among the clients of a network and no one else can access them.

Finally, dealing with contingencies is focused not only on UAS emergencies and systems failures but also on other off-nominal situations. Mainly, the UAS operator is responsible to effectively deal with emergencies and maintain the safe operation of the UAS or safely terminate the flight if it becomes uncontrolled. The provision of necessary information and assistance to UAS operators (similar to CORUS) is available through the USS network with the involvement of the service providers and the FAA. (Ref. [15]).

Ultimately, it is worth mentioning that service provision (usually, location and operation-oriented), SA assurance, oversight by the respective authorities and the ATC, where applicable, and successfully confronting contingencies and unexpected situations are only available through effective and timely data sharing among the UAS stakeholders. Same is the case in the CORUS.

\section{SORA Methodology (EU)}

The 'Specific' Operations Risk Assessment (SORA) methodology is developed by JARUS and describes the process, rules and prerequisites for conducting an operational risk assessment. This methodology is incorporated in the CORUS and accepted by EASA as the main tool for safety risk assessment and obtaining approval for 'Specific' category UAS operations ${ }^{9}$.

The SORA methodology aims at assuring that the proposed operation, during its duration, will remain under control and conducted safely. Should part of the operation go out of control then the required mitigation measures will be applied in order to continuously maintaining the operation under control. In the case of losing the control of the operation then emergency procedures are to be applied according to the operational plan and the Emergency Response Plan (ERP) in particular. The SORA methodology takes into account the operating airspace in order to define how the respective operation will remain under control. Regarding the operating airspace, the outcome of applying the SORA is depicted in Fig. 4. (Ref. [8]).

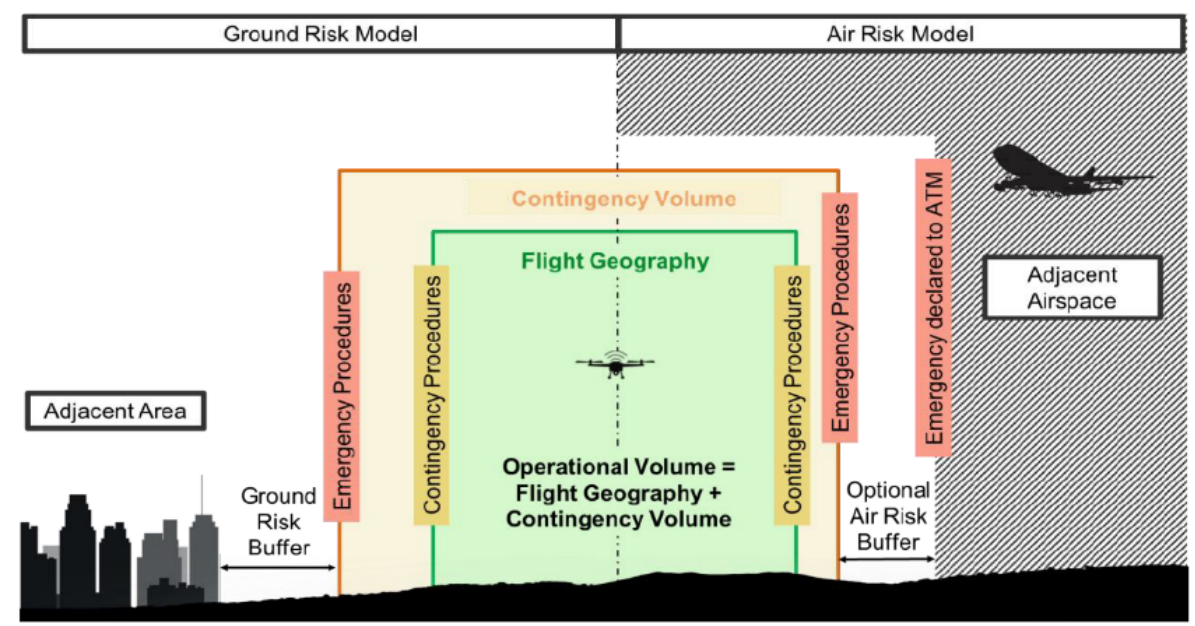

Fig. 4 Graphical representation of the SORA semantic model.

The steps of the SORA process are shown in Fig. 5. (Ref. [8]). Initially, the intrinsic Ground Risk Class (GRC) needs to be determined by correlating the operational scenarios (or the most suitable for the intended operation if not in the list) with the characteristics of the UAS (i.e., dimensional characteristics and expected kinetic energy). The outcome is a value from 1 to 7 which corresponds to the initial GRC. Next, in order to determine the final GRC, the initial GRC is combined with three mitigation measures. All of these mitigations should be applied for determining

${ }^{9}$ UAS operators holding a Light UAS operator Certificate (LUC) are not required to perform SORA and submit an operational declaration. (Ref. [8]). 
the final GRC. It should be mentioned that these mitigations are basically, strategic. If the final GRC is greater than 7 then the respective operation is not supported by the SORA process.

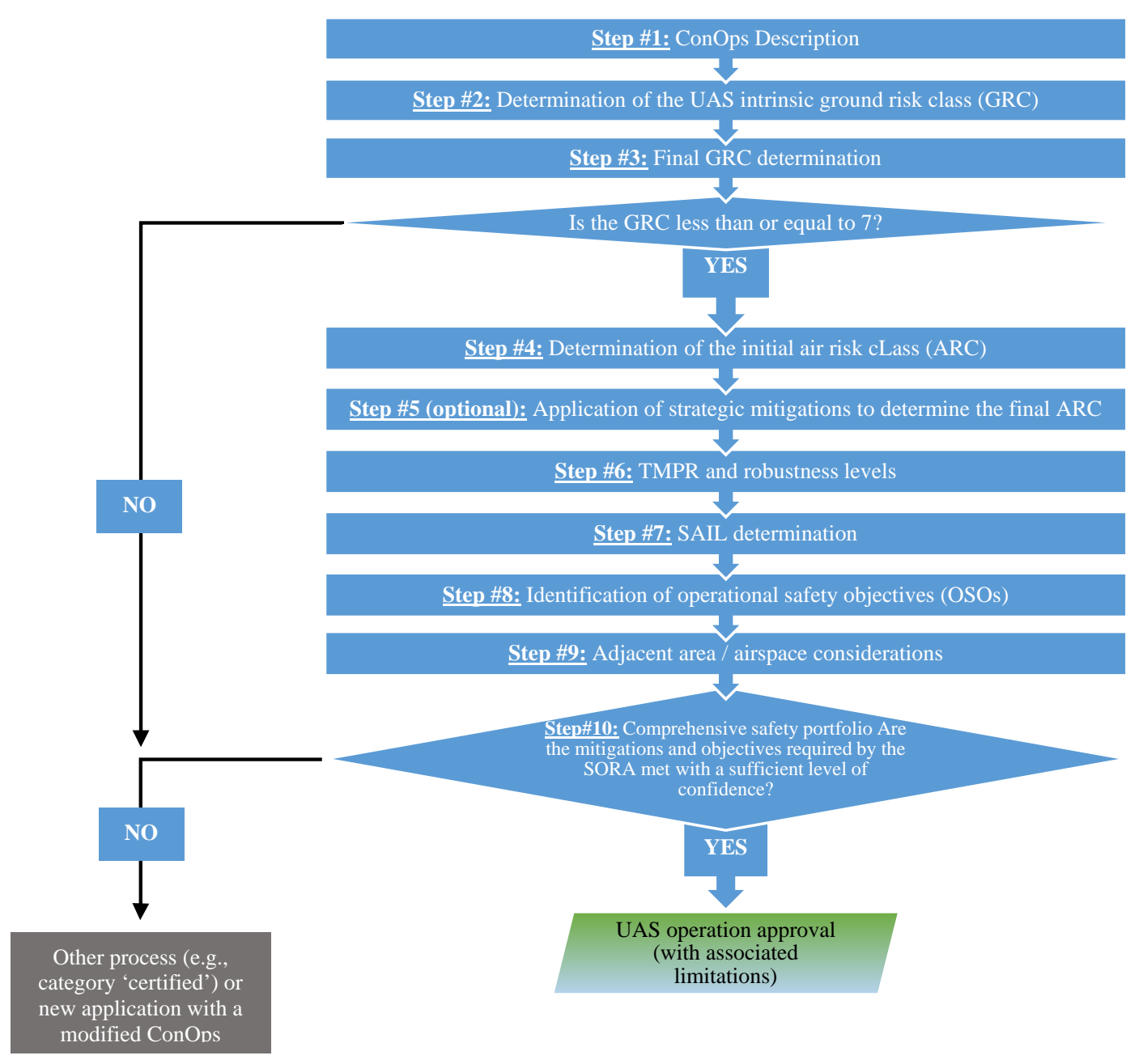

Fig. 5 The SORA process.

Next, the operator needs to determine the initial Air Risk Class (ARC). For determining the ARC, the SORA process takes into account the different types of airspace defined by the altitude, vicinity to an airport or heliport, controlled or uncontrolled, rural or urban environment, and segregated versus typical, and the risk of collision with a manned aircraft. The ARC is determined from 'a' to 'd' (e.g., ARC-b) meaning that the risk of a MAC is greater as the class advances from ARC-a to ARC-d. After the initial determination of the ARC, there is an optional step, Step \#5, where the operator may decrease the ARC by applying strategic mitigations, only. Then the residual ARC needs to be determined. This happens in Step \#6 where the respective Tactical Mitigation Performance Requirements (TMPRs) and robustness levels are correlated to each ARC. TMPR takes into account the risk of a MAC and defines the level of performance in avoiding a collision with a manned aircraft. The assignment for each ARC is shown in Table 4. (Ref. [8]). In VLOS operations the TMPR is met through the SAA function performed by the RIC. 
Table 4 TMPRs and TMPR level of robustness assignment.

\begin{tabular}{|c|c|c|}
\hline Residual ARC & TMPRs & TMPR level of robustness \\
\hline ARC-d & High & High \\
\hline ARC-c & Medium & Medium \\
\hline ARC-b & Low & Low \\
\hline ARC-a & No requirement & No requirement \\
\hline
\end{tabular}

Conversely, in BVLOS operations, TMPRs are based on the DAA function because the RIC is not always in visual contact with the UAS. So, TMPRs are applied to each sub-function of the DAA, which are the following: detect, decide, command, execute, and feedback loop. (Ref. [8]). An indicative example is shown in Table 5 concerning the 'execute' phase of DAA. (Ref. [8]). The last phase of Step \#6 is determining the robustness level for TMPR/ARC level. The respective assurance and integrity objectives are shown in Table 6. (Ref. [8]).

Table 5 TMPR for 'Execute' and the requirements for each TMPR level.

\begin{tabular}{|c|c|c|c|c|c|c|}
\hline & \multirow[b]{2}{*}{ Function } & \multicolumn{5}{|c|}{ TMPR Level } \\
\hline & & VLOS & $\begin{array}{c}\text { No } \\
\text { Requirement } \\
\text { (ARC-a) }\end{array}$ & $\begin{array}{c}\text { Low } \\
\text { (ARC-b) }\end{array}$ & $\begin{array}{l}\text { Medium } \\
\text { (ARC-c) }\end{array}$ & $\begin{array}{c}\text { High } \\
\text { (ARC-d) }\end{array}$ \\
\hline 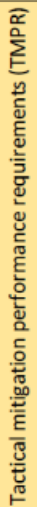 & Execute & 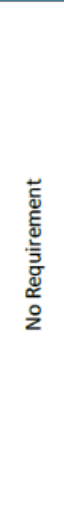 & 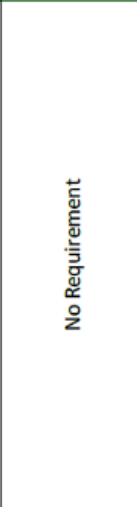 & $\begin{array}{l}\text { UAS descending to an altitude not higher than the } \\
\text { nearest trees, buildings or infrastructure or } \leq 60 \\
\text { feet AGL is considered sufficient. } \\
\text { The aircraft should be able to descend from its } \\
\text { operating altitude to the 'safe altitude' in less than } \\
\text { a minute. }\end{array}$ & $\begin{array}{l}\text { Avoidance may rely on vertical and horizontal } \\
\text { avoidance manoeuvring and is defined in } \\
\text { standard procedures. Where horizontal } \\
\text { manoeuvring is applied, the aircraft shall be } \\
\text { demonstrated to have adequate performance, } \\
\text { such as airspeed, acceleration rates, } \\
\text { climb/descend rates and turn rates. The following } \\
\text { are suggested minimum performance criteria: }{ }^{10} \\
\text { - Airspeed: } \geq 50 \text { knots } \\
\text { - Rate of climb/descend: } \geq 500 \mathrm{ft} / \mathrm{min} \\
\text { - Turn rate: } \geq 3 \text { degrees per second }\end{array}$ & $\begin{array}{l}\text { A system } \\
\text { meeting RTCA } \\
\text { SC- } 228 \text { or } \\
\text { EUROCAE WG- } \\
105 \\
\text { MOPS/MASPS } \\
\text { (or similar) } \\
\text { and installed in } \\
\text { accordance } \\
\text { with applicable } \\
\text { requirements. }\end{array}$ \\
\hline
\end{tabular}

In Step \#7 the Safety Assurance and Integrity Level (SAIL) is determined. According to Ref. [8], 'the SAIL represents the level of confidence that the UAS operation will remain under control.' The SAIL can take one of the six values (i.e., I to VI) and is a function of the final GRC, and the residual ARC. It is assigned to a particular ConOps and combined with the OSOs in the context of the respective ConOps drives the level of performance and mitigation measures that an UAS operator should have in place. Table 7 shows the SAIL determination. (Ref. [8]).

Next, in Step \#8, the SAIL is combined with the OSOs in order to determine the level of robustness for each OSO. There are twenty-four recommended OSOs. However, a competent authority may develop and apply additional or different OSOs and thus request the respective level of performance from the UAS operator. Each OSO is characterized by a different level of robustness which can be Optional (O), Low (L), Medium (M) or High (H). Table 8 shows an indicative example of three OSOs. (Ref. [8]). Table 9 shows, for a specific OSO, how the different levels of robustness could be achieved. (Ref. [8]).

In Step \#9, the risks that an unmanned operation could pose to adjacent areas are addressed. For this paper the risks addressed in this step will be considered as the responsibility of the respective adjacent UAS operator and the UAS operator in a certain area will have to assure that the UAS will not exceed the limits of that area. Finally, Step \#10 closes the loop of the SORA process reassuring that the operator has in place all the required mitigations as they have been derived from the SORA and is able to address any other risk associated with the safe and controlled execution of the intended operation. 
Table 6 TMPR integrity and assurance levels.

\begin{tabular}{|c|c|c|c|c|c|}
\hline & & $\begin{array}{c}\text { TMPR: N/A } \\
\text { (ARC-a) }\end{array}$ & $\begin{array}{l}\text { TMPR: Low } \\
\text { (ARC-b) }\end{array}$ & $\begin{array}{l}\text { TMPR: Medium } \\
\text { (ARC-c) }\end{array}$ & $\begin{array}{l}\text { TMPR: High } \\
\text { (ARC-d) }\end{array}$ \\
\hline \multirow{3}{*}{$\begin{array}{l}\text { Level of } \\
\text { integrity }\end{array}$} & Criteria & $\begin{array}{l}\text { Allowable loss of } \\
\text { function and } \\
\text { performance of the } \\
\text { Tactical Mitigation } \\
\text { System: }<1 \text { per } 100 \\
\text { Flight Hours } \\
\text { (1E-2 Loss/FH) }\end{array}$ & $\begin{array}{l}\text { Allowable loss of } \\
\text { function and } \\
\text { performance of the } \\
\text { Tactical Mitigation } \\
\text { System: }<1 \text { per } 100 \\
\text { Flight Hours } \\
\text { (1E-2 Loss/FH) }\end{array}$ & $\begin{array}{c}\text { Allowable loss of } \\
\text { function and } \\
\text { performance of the } \\
\text { Tactical Mitigation } \\
\text { System: }<1 \text { per } 1000 \\
\text { Flight Hours } \\
\text { (1E-3 Loss/FH) }\end{array}$ & $\begin{array}{c}\text { Allowable loss of } \\
\text { function and } \\
\text { performance of the } \\
\text { Tactical Mitigation } \\
\text { System: }<1 \text { per } 100000 \\
\text { Flight Hours } \\
\text { (1E-5 Loss/FH) }\end{array}$ \\
\hline & $\begin{array}{c}\text { Comments / } \\
\text { Notes }\end{array}$ & $\begin{array}{l}\text { The requirement is } \\
\text { considered to be met } \\
\text { by commercially } \\
\text { available products. } \\
\text { No quantitative } \\
\text { analysis is required. }\end{array}$ & $\begin{array}{l}\text { The requirement is } \\
\text { considered to be met } \\
\text { by commercially } \\
\text { available products. } \\
\text { No quantitative } \\
\text { analysis is required. }\end{array}$ & $\begin{array}{c}\text { This rate is } \\
\text { commensurate with } \\
\text { a probable failure } \\
\text { condition. These } \\
\text { failure conditions are } \\
\text { anticipated to occur } \\
\text { one or more times } \\
\text { during the entire } \\
\text { operational life of } \\
\text { each aircraft. }\end{array}$ & $\begin{array}{l}\text { A quantitative analysis is } \\
\text { required. }\end{array}$ \\
\hline & & $\begin{array}{c}\text { TMPR: N/A } \\
\text { (ARC-a) }\end{array}$ & $\begin{array}{l}\text { TMPR: Low } \\
\text { (ARC-b) }\end{array}$ & $\begin{array}{l}\text { TMPR: Medium } \\
\text { (ARC-c) }\end{array}$ & $\begin{array}{l}\text { TMPR: High } \\
\text { (ARC-d) }\end{array}$ \\
\hline \multirow[t]{2}{*}{$\begin{array}{l}\text { Level of } \\
\text { assurance }\end{array}$} & Criteria & N/A & $\begin{array}{c}\text { The operator } \\
\text { declares that the } \\
\text { tactical mitigation } \\
\text { system and } \\
\text { procedures will } \\
\text { mitigate the risk of } \\
\text { collisions with } \\
\text { manned aircraft to an } \\
\text { acceptable level. }\end{array}$ & $\begin{array}{l}\text { The operator } \\
\text { provides evidence } \\
\text { that the tactical } \\
\text { mitigation system } \\
\text { will mitigate the risk } \\
\text { of collisions with } \\
\text { manned aircraft to an } \\
\text { acceptable level. }\end{array}$ & $\begin{array}{l}\text { The evidence that the } \\
\text { tactical mitigation } \\
\text { system will mitigate the } \\
\text { risk of collisions with } \\
\text { manned aircraft to an } \\
\text { acceptable level is } \\
\text { verified by a competent } \\
\text { third party. }\end{array}$ \\
\hline & $\begin{array}{c}\text { Comments / } \\
\text { Notes }\end{array}$ & $N / A$ & $N / A$ & $N / A$ & $N / A$ \\
\hline
\end{tabular}

Table 7 SAIL determination.

\begin{tabular}{|c|c|c|c|c|}
\hline \multicolumn{4}{|c|}{ SAIL determination } \\
\hline & & \multicolumn{2}{|c|}{ Residual ARC } \\
\hline Final GRC & a & b & c & d \\
\hline$\leq 2$ & I & II & IV & VI \\
\hline 3 & II & II & IV & VI \\
\hline 4 & III & III & IV & VI \\
\hline 5 & IV & IV & IV & VI \\
\hline 6 & V & V & V & VI \\
\hline 7 & VI & VI & VI & VI \\
\hline$>7$ & & Category Coperation & \\
\hline
\end{tabular}


Table 8 Recommended OSOs (not full list).

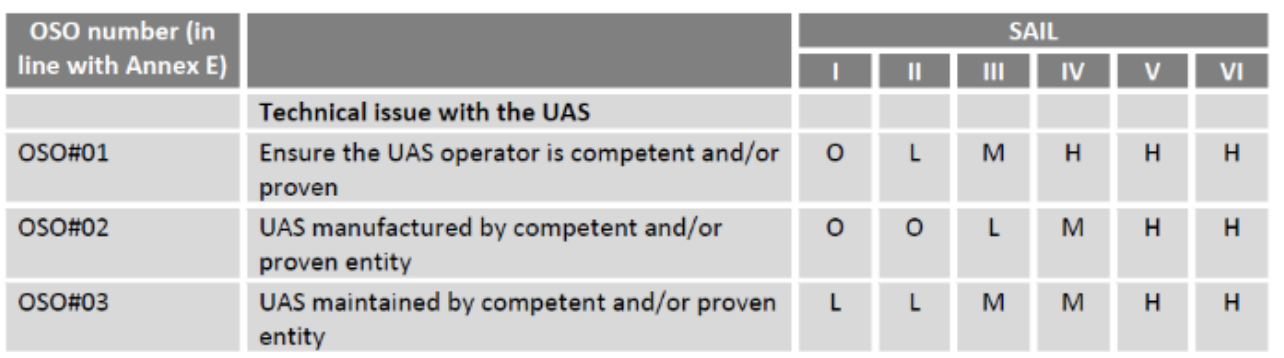

Table 9 OSO\#1 Different robustness levels and requirements.

\begin{tabular}{|c|c|c|c|c|}
\hline \multirow{2}{*}{\multicolumn{2}{|c|}{ TECHNICAL ISSUE WITH THE UAS }} & \multicolumn{3}{|c|}{ Level of integrity } \\
\hline & & Low & Medium & High \\
\hline \multirow{2}{*}{$\begin{array}{l}\text { OSO \#01 } \\
\text { Ensure that } \\
\text { the UAS } \\
\text { operator is } \\
\text { competent } \\
\text { and/or } \\
\text { proven }\end{array}$} & Criteria & $\begin{array}{l}\text { The applicant is knowledgeable of the UAS } \\
\text { being used and as a minimum has the } \\
\text { following relevant operational procedures: } \\
\text { checklists, maintenance, training, } \\
\text { responsibilities, and associated duties. }\end{array}$ & $\begin{array}{l}\text { Same as low. In addition, the applicant has an } \\
\text { organisation appropriate }{ }^{1} \text { for the intended } \\
\text { operation. Also, the applicant has a method to } \\
\text { identify, assess, and mitigate the risks associated } \\
\text { with flight operations. These should be consistent } \\
\text { with the nature and extent of the operations } \\
\text { specified. }\end{array}$ & Same as medium. \\
\hline & Comments & $N / A$ & $\begin{array}{l}{ }^{1} \text { For the purpose of this assessment, 'appropriate' } \\
\text { should be interpreted as commensurate } \\
\text { with/proportionate to the size of the organisation } \\
\text { and the complexity of the operation. }\end{array}$ & $N / A$ \\
\hline
\end{tabular}

\begin{tabular}{|c|c|c|c|c|}
\hline \multirow{2}{*}{\multicolumn{2}{|c|}{ TECHNICAL ISSUE WITH THE UAS }} & \multicolumn{3}{|c|}{ Level of assurance } \\
\hline & & Low & Medium & High \\
\hline \multirow[t]{2}{*}{$\begin{array}{l}\text { OSO \#01 } \\
\text { Ensure that } \\
\text { the UAS } \\
\text { operator is } \\
\text { competent } \\
\text { and/or proven }\end{array}$} & Criteria & $\begin{array}{l}\text { The elements delineated in the level of } \\
\text { integrity are addressed in the ConOps. }\end{array}$ & $\begin{array}{l}\text { Prior to the first operation, a competent } \\
\text { third party performs an audit of the } \\
\text { organisation }\end{array}$ & $\begin{array}{l}\text { The applicant holds an organisational } \\
\text { operating certificate or has a } \\
\text { recognised flight test organisation. } \\
\text { In addition, a competent third party } \\
\text { recurrently verifies the UAS operator's } \\
\text { competences. }\end{array}$ \\
\hline & Comments & N/A & N/A & $N / A$ \\
\hline
\end{tabular}

It must be noted that the SORA methodology does not cover UAS operations of the 'Certified' category. Moreover, it covers only the safety risks that UAS operations pose to manned aircraft (e.g., MAC or loss of adequate separation) and to people and properties on ground. These risks are assessed and mitigated in the context of the operating airspace. In addition, it does not cover the risks that a UAS operation may pose to another similar operation (e.g., MAC between two UAS). Also, out of the scope of the SORA are the safety risks related but not limited to weather, terrain, bird strikes, transferring of people and carrying weapons.

\section{Civil Aviation Publication (CAP) 722 (UK)}

The Ref. [37] outlines that an operational authorization is required for 'Specific' category operations. Regarding these operations, in Ref. [37], it is stated that 'UAS used in the 'Specific' category are not subject to any particular classification. Their technical standards are dependent on the proposed type of operation and its associated risk assessment.' So, the UAS operator needs to provide a description of the intended operation and a risk assessment for the respective operation. If the operation is not covered by a Pre-defined Risk Assessment (PDRA) ${ }^{10}$, the operator must conduct a safety risk assessment according to Ref. [38].

In Ref. [38] it is highlighted that 'each Safety Risk should be at a TOLERABLE and ALARP'11 level to be acceptable to the Civil Aviation Authority (CAA).' Ref. [38] describes the safety risk assessment as a three-stage procedure. These stages are the following: $1^{\text {st }}$ Safety Risk Assessment Process (SRAP), $2^{\text {nd }}$ Safety Risk Assurance, and $3^{\text {rd }}$ Safety Risk Summary Statement. (Ref. [38]). In Stage 2 the Ref. [38] makes provision for the Safety Risk Assurance.

${ }^{10}$ PDRA - A risk assessment that has been already conducted based on certain assumptions (e.g., typical kinetic energy of up to 34 kilo Joule, BVLOS, $500 \mathrm{ft}$ maximum altitude, etc.) and generally expedites the operational approval. ${ }^{11}$ ALARP - As Low As Reasonably Practicable: The lowest achievable level in which a risk can be reduced based on the available resources (i.e., money, and time). Further reduction attempt leads to disproportionate spending of resources in relation to the actual decrease in risk. Ref. [38]. 
This is a self-assessment conducted by the operator that actually provides evidence and proofs how the desirable outcome has been achieved in Stage 1. For example, according to Ref. [38], Stage 2 describes the methodology followed by the operator in Stage 1. In Stage 3 the Safety Risk Summary Statement finalizes the Safety Risk Assessment process. This stage summarizes the Operating Safety Case in which the whole process was based. In Ref. [38] the safety risk is initially categorized using three escalated levels which are the Unacceptable, Review, and Acceptable. (Ref. [38]). The respective definitions are shown below:

- $\quad$ Unacceptable - The Safety Risk is not tolerable and mitigation measures are required to reduce the Safety Risk to a tolerable level.

- $\quad$ Review - The Safety Risk is tolerable, but only just. The risk should be reviewed with appropriate frequency to ensure that it remains so.

- $\quad$ Acceptable - The safety Risk is tolerable and should be reviewed with appropriate frequency to ensure that it remains so.

Besides the assessment of risk tolerability, the operator must provide an assessment of ALARP. Then similar to the SORA methodology, the UK CAA denotes that the safety mitigations applied should be assessed for their robustness. The operator is responsible for the defining and proving the robustness. According to Ref. [38]), robustness of safety mitigations is a function of Performance and Integrity. Performance is the outcome that a mitigation may have on a safety risk and Integrity is the assurance that this outcome will be delivered.

As far as the collision avoidance is concerned, DAA requirements have been established as well as separation services that are provided by the ATC in IFR and VFR flights. As described in Ref. [37], routine BVLOS operations in non-segregated airspace are required to be equipped with DAA capabilities unless it can be assured that the operation will not pose any hazard to other aircraft. The flight rules, type of UAS and airspace class differentiate the level of DAA capability that should be demonstrated. Table 10 provides a summary of how separation between aircraft may be achieved, according to the CAP 722 .

Finally, it is worthwhile to mention the RID requirements that the UK CAA has set. RID has been established not only to raise SA between airspace users but also for oversight and law enforcement purposes. RID may take the form of Direct RID and/or Network RID. (Ref. [37]). The main difference between these RID options is the transmission of UAS information within a network and respective users or not. It is also highlighted that by December 2, 2021, all UAS operating in the 'Specific' category must be equipped with RID systems.

Table 10 Collision avoidance provisions.

\begin{tabular}{|c|c|c|c|c|}
\hline & & \multicolumn{2}{|r|}{ Controlled Airspace (Classes A-E) } & Class $\mathrm{G}$ airspace \\
\hline & & Required equipment & Services provided/Actions required & \\
\hline \multirow{4}{*}{$\begin{array}{l}\text { Type } \\
\text { of } \\
\text { flight }\end{array}$} & \multirow{4}{*}{ IFR } & \multirow{4}{*}{$\begin{array}{l}\text { A Collision } \\
\text { Avoidance capability } \\
\text { will be required. }\end{array}$} & $\begin{array}{l}\text { ATC separates from other traffic (although in Class } \\
\text { D and E, the pilot of a conflicting VFR flight holds } \\
\text { the separation responsibility). }\end{array}$ & \\
\hline & & & $\begin{array}{l}\text { As for manned aviation, a collision avoidance } \\
\text { capability is required in case the 'normal' separation } \\
\text { provision fails. }\end{array}$ & \\
\hline & & & $\begin{array}{l}\text { If the flight is conducted wholly within controlled } \\
\text { airspace where the operation of a transponder is } \\
\text { mandatory, then a collision avoidance capability that } \\
\text { is cooperative (e.g., ACAS) would be acceptable. }\end{array}$ & \\
\hline & & & $\begin{array}{l}\text { If there is any possibility that the UAS will/might } \\
\text { leave controlled airspace and enter non-segregated } \\
\text { Class G airspace during the flight (including in an } \\
\text { emergency), then the collision avoidance capability } \\
\text { must be 'non-cooperative', unless there are other } \\
\text { airspace measures in place that would still allow a } \\
\text { cooperative system to be used. }\end{array}$ & \\
\hline
\end{tabular}




\begin{tabular}{|c|c|c|c|c|}
\hline & & \multicolumn{2}{|c|}{ Controlled Airspace (Classes A-E) } & Class G airspace \\
\hline & & Required equipment & Services provided/Actions required & \\
\hline $\begin{array}{l}\text { Type } \\
\text { of } \\
\text { flight }\end{array}$ & VFR & $\begin{array}{c}\text { A Separation } \\
\text { Assurance/Traffic } \\
\text { Avoidance capability } \\
\text { and } \\
\text { a Collision } \\
\text { Avoidance capability } \\
\text { will be required. }\end{array}$ & $\begin{array}{c}\text { The remote pilot is the separator for all conflicts, } \\
\text { with the same responsibilities as the pilot of a } \\
\text { manned aircraft. }\end{array}$ & $\begin{array}{l}\text { Any flight in } \\
\text { G class: } \\
\text { Same as VFR }\end{array}$ \\
\hline
\end{tabular}

\section{E. Avoiding Collisions}

Avoiding collisions with the terrain and collisions between UAS and manned aircraft as well as between UAS is the ultimate priority of the ConOps and the regulations mentioned before. Another critical aspect is the oversight in case of unruly UAS operators or in cases that a UAS may encounter an emergency situation. In both cases a UAS may be unable to comply with the flight rules for the respective airspace or the ATC instructions and thus compromising safety. Oversight is a part of safety assurance. Effective oversight accompanied by the ability to intervene can result in avoiding loss of adequate separation and finally collisions.

Unlike manned aviation, UAS RIC may not always be to talk to the ATC or other aircraft on the radio in order to verify and exchange information regarding flight. At the same time every aircraft operating in a certain area need to be aware of other aircraft in the vicinity. The most indicative and time-wise method to increase and maintain everyone's SA is flight data sharing. Data sharing promotes safety assurance and safety oversight. Even in the case of non-cooperative traffic, the cooperative traffic that receives data for the non-cooperative one, can make the required changes in flight in order to maintain adequate separation. In the case that the non-cooperative traffic does not transmit any data then the separation should be maintained using non-cooperative means like radar. Some indicative examples are shown in Table 11. Ref. [7], [26]. Regarding airspace integration cooperative and non-cooperative traffic will enjoy different level of integration. UAS with no detection systems (cooperative or non-cooperative) should be considered as non-cooperative air traffic.

Table 11 Cooperative and Non-Cooperative systems.

\begin{tabular}{lll}
\hline & Cooperative & Non-Cooperative \\
\hline & TCAS & Electro-Optical (EO) Cameras \\
\cline { 2 - 3 } ETCAS & High Resolution Cameras \\
\cline { 2 - 3 } Respective Systems & GDL-84 ADS-B ${ }^{12}$ Datalink & Television (TV) Cameras \\
\cline { 2 - 3 } & GDL-88 ADS-B Datalink System & Infrared (IR) Sensors \\
\cline { 2 - 3 } & Lynx NGT-9000 & On-board Radars \\
\hline & & $\begin{array}{l}\text { Light Detection And Ranging (LIDAR) - } \\
\text { Laser based detection system }\end{array}$ \\
\hline
\end{tabular}

${ }^{12}$ ADS-B - Automatic Dependent Surveillance Broadcast. ADS-B is defined as a system that uses GPS technology to determine an aircraft's location, airspeed and other data, and broadcasts that information to a network of transceivers, which relays the data to ATC displays. (Ref. [16]). The broadcasting aircraft as well as the receiver (aircraft or ATC) must be also equipped with ADS-B in order for the messages to be received and handled. 


\section{Manned Aviation Legacy}

\section{A. Safety Assurance}

According to Ref. [19], the Safety Assurance component consists of three elements being the Safety performance monitoring and measurement, the Management of change, and the Continuous improvement of the SMS. While all the three are linked to some extent to the safety risks of an operation, they do not correspond to the same stage of an operation. In terms of conducting an operation (i.e., fly an aircraft for a certain purpose) the element that is mostly involved is the Safety performance and monitoring. The other two are not so operation-centric. Changes in the staffing levels of an organization, changes in the existing technology or changes in the organizational structure could require management and constitute factors that may affect the operation itself. For example, in a commercial airline the safety of a flight should not be compromised due to a change in the operating environment (e.g., the introduction of a new route). Regarding the Continuous improvement of the SMS, it actually answers the question of how well the other two elements are being implemented. Focusing on Safety performance monitoring and measurement, in Ref. [19], it is stated that 'to verify the safety performance and validate the effectiveness of safety risk controls requires the use of a combination of internal audits and the establishment and monitoring of SPIs.' Internal audits are performed in order to verify and assess the level in which regulations and procedures are followed and also to check the effectiveness of safety risk controls and mitigating actions. As far as the SPIs are concerned, they are interconnected to the SPTs and the safety objectives that are declared by an organization or an operator. In more detail, an operator sets a safety objective and then sets a SPT to monitor the progress towards achieving that particular safety objective. In parallel, there may be one or more SPIs, related to a SPT, aid to collecting data and monitoring performance. Based on SPIs, alert levels can be set that are linked to the level of achievement of a SPT. Alert levels can trigger actions in order to reach again a former achieved SPT or highlight the necessity for further actions towards achieving a SPT. Consider the following example:

An airline sets the Safety Objective of achieving zero fatal accidents during all flights for a ten-year period. For a particular category of fatal accidents, the MACs, the same objective is set. Bearing in mind that MACs can be the result of 1) loss of adequate separation between conflicting aircraft, 2) delayed pilot reaction, 3) pilots not complying with certain procedures, 4) aggressive and/or non-standard maneuvers, just to name some, the respective SPTs are set which are 1) maintaining at least $1000 \mathrm{ft}$ vertical separation and 15 nautical miles from other aircraft (based on Ref. [21]), 2) set a certain maximum reaction time, for example 3 seconds, 3) pilot incompliance with ATC instructions, and 4) set standard limits for every aircraft maneuver. Focusing on SPT 1 the respective SPI could be the frequency that the respective limits were violated in a number of 100 flights.

Safety performance is ultimately linked to safety risk management. Setting and monitoring SPTs and SPIs trigger actions required for safety risk mitigation. Risk is regarded by ICAO to be a function of probability and severity. Probability expressed in frequency and severity expressed in the catastrophic level of the effects form the tolerability ${ }^{13}$ of a risk. According to ICAO, the risks that fall into the Intolerable region need to be further mitigated and if further mitigation does not achieve to put these risks at least in the Tolerable region then the respective operation or activity should not continue. The main mitigation strategies that are mentioned in Ref. [19], are the following:

1) Avoidance: The operation or activity is cancelled or avoided because the safety risk exceeds the benefits of continuing the activity, thereby eliminating the safety risk entirely.

2) Reduction: The frequency of the operation or activity is reduced, or action is taken to reduce the magnitude of the consequences of the safety risk.

3) Segregation: Action is taken to isolate the effects of the consequences of the safety risk or build in redundancy to protect against them.

\section{B. Certification}

In order to achieve the desired level of performance and thus maintaining flight safety aircraft and flight crew have to be certified. Certification assures that a minimum level of standards has been achieved and respectively the aircraft and the staff involved in flight execution are certified. In the EU staff certification is described in detail by EASA, in the USA by FAA and similar is the case around the world. For example, as described in Ref. [10], the basic theoretical knowledge for a Commercial Pilot License (CPL) is shown in Table 12. Respective CS apply for other staff like aircraft engineers and air traffic controllers.

\footnotetext{
${ }^{13}$ Probability expressed in frequency and valued from 1 (lowest probability) to 5 (highest probability). Severity expressed in terms of the catastrophic level and valued from A (catastrophic effects) to E (negligible effects).
} 
Table 12 CPL Theoretical Knowledge Requirements.

\begin{tabular}{cc}
\hline Air Law & Human Performance \\
\hline $\begin{array}{c}\text { Aircraft General Knowledge- } \\
\text { Airframe/Systems/Powerplant }\end{array}$ & Meteorology \\
\hline Aircraft General Knowledge-Instrumentation & General Navigation \\
\hline Mass and Balance & Radio Navigation \\
\hline Performance & Operational Procedures \\
\hline Flight Planning and Monitoring & \\
\hline
\end{tabular}

In regard to aircraft certification, it could be considered as an add-on in the existing airworthiness. Based on the type of aircraft (e.g., commercial, military cargo or fighter, helicopter, etc.,) there are certain airworthiness standards that are applied during the design and the construction phases. Next based on the exact type of the operation, the operating airspace or directives that may be oriented from the operator or the authority controlling the airspace, additional certification may be required in order to assure that the aircraft or an aircraft system will operate as requested. An indicative example is the TCAS that requires certification after its installation on the aircraft and also certification pertain to pilots as an assurance that they know how to use it while in-flight.

\section{Performance-based Operation}

The Performance-based Operation (PBO) concept was initially introduced by ICAO in the form of Performancebased Navigation (PBN) and was related to navigation only. As mentioned in Ref. [22], the PBN concept relies on the accuracy, integrity, continuity, and functionality of aircraft navigation systems and also to the relevant pilot training and procedural adherence. Amongst the advantages of PBN that are mentioned in Ref. [23] the most critical could be considered the reduce of vertical separation minima, the evolution of new routes (shorter and thus burning less fuel or avoiding congested areas), easier air traffic management, and the increment of aircraft that can operate in certain areas. Today, different rules are applied to aircraft with certain contemporary navigation performance in contrast to aircraft with lower navigation capabilities. Moreover, in some airspace classes there are certain navigation criteria that need to be fulfilled otherwise the operation may not be approved. Regarding the advantages of PBN, in Ref. [12], it is mentioned that PBN can result in greater level of safety, capacity, and efficiency through the optimization of the air traffic routes and instrument approaches.

Like navigation, collision avoidance is another aspect of PBO. Collision avoidance systems like TCAS have become mandatory aircraft equipment in most national airspaces. In the EU, all commercial aircraft must be equipped with TCAS although different procedures may be followed in the event that system becomes inoperable. Yet, there are aircraft without TCAS like many military and general aviation aircraft. Indeed, according to Ref. [20], a manned aircraft weighing less than $5,700 \mathrm{~kg}$ or carrying less than 19 passengers does not need to be equipped with TCAS. In addition, there may be parts of an airspace where the use of a transponder is mandatory (Mandatory Transponder Zones). In the future, similar mandatory zones may be created like mandatory ADS-B zones.

Inferentially, the PBO concept results in aircraft being able or unable to operate in certain environments and when they do operate different rules may be applied in comparison to other less capable aircraft. This could be assumed as a type of segregation (performance-based) considering that an operation may not be approved or be amended due to performance factors.

\section{Unmanned Aviation Perspective}

\section{A. UAS Certification}

Based on the research in which this paper is based, it seems that no international CS exist for the 'Specific' category UAS. Countries develop standards on a case-by-case concept that is the kind of operation each UAS is intended for. Below the case of the EU and the USA are briefly described.

1) $\mathrm{EU}$

Currently, the certification of unmanned aircraft that is conducted according to the respective manned aircraft standards, is for the certification of UAS that are used for operations falling into the 'Certified' category. This certification is based on the Commission Regulations ${ }^{14}$ (EU) No 748/2012, (EU) 2015/640, and No 1321/2014. (Ref.

${ }^{14}$ All the three regulations cover aircraft airworthiness rules and specifications. 
[8]). According to the same rules the 'Specific' category UAS need an operational authorization and a risk assessment in order to commence operations. Certification of certain aircraft systems and other products installed in these UAS serve only the purpose of further risk mitigation. In this case, certification is an additional assurance that the system will operate safely. Regarding certification standards, based on Ref. [11] the use of manned aircraft CS is accepted and some 'Special Conditions' (SC) that request additional attention and thus development of CS could be the following:

- $\quad$ Emergency Recovery Capability

- $\quad$ Command and Control Link

- Level of Autonomy

- Human Machine Interface

- Control station

- Due to type of operation

- $\quad$ System Safety Assessment

In March 2021, EASA published guidelines related to 'Design Verification' for UAS operations that are classified based on the SORA, as SAIL III and IV. According to Ref. [9], for UAS operations assessed as SAIL V \& VI EASA will issue type-certificate or restricted type-certificate according to the Commission Regulation No 748/2012 and for UAS operations assessed as SAIL III \& IV EASA will perform a design verification covering one or more of the following topics: 1. the full design of the UAS, 2. the mitigation means linked with the design, and 3. the enhanced containment function. It should be noted that in Ref. [9] it is pointed out that EASA is competent to perform the aforementioned verification while the member states are competent to assess the competency of those remote pilots that will get involved in the UAS operations SAIL III \& IV.

\section{2) USA}

The Part 107 of the $14^{\text {th }}$ Code of Federal Regulation covers the operations and the respective airworthiness standards of the UAS weighing less than 55 pounds (approximately $25 \mathrm{~kg}$ ), categorized as small UAS (sUAS). Part 107 prohibits sUAS operations at night and operations over people. FAA (2016). Recently, on April 2021, FAA published the 'Operations Over People rule'. (Ref. [13]). According to that rule, sUAS under the Part 107 may operate at night, over people and from moving vehicles. Mainly, according to the 'Operations Over People rule', the sUAS category is further divided into four categories. The rule is performance-based and takes into account the total energy that will be released upon impact of a sUAS on the ground or on people. For bigger UAS, FAA also applies CS similar to those applied on manned aircraft.

\section{B. Remote Pilot Training}

In terms of aircraft handling and executing basic aviating tasks like takeoff, approach, and landing as well as navigating inside the airspace, a remote pilot training could not be much different than the respective training of a manned aircraft pilot. According to Ref. [24], remote pilots shall be trained and licensed in accordance with Annex $1^{15}$ and the licensing and training requirements will be similar to those of manned aviation and will include both the aeronautical knowledge and operational components. Table 13 shows some basic competencies that a remote pilot should have as described in Ref. [25]. Today, what should be added is the skill of maintaining high SA for other aircraft, manned and unmanned, in the vicinity of the operating airspace. Apart from skill, this is also a technologydriven ability that needs to be provided to the RIC. Nevertheless, remote pilots should be also trained in this activity.

Table 13 Remote Pilot Competencies.

\begin{tabular}{c|c}
\hline Subjects of knowledge & Practical skills \\
\hline Air law; & Recognize and manage threats and errors; \\
\hline RPAS general knowledge; & $\begin{array}{c}\text { Operate the RPA within its limitations or those } \\
\text { limitations imposed by regulation; }\end{array}$ \\
\hline Flight performance, planning, and loading; & $\begin{array}{c}\text { Complete all maneuvers with smoothness and } \\
\text { accuracy; }\end{array}$ \\
\hline Human performance; & Exercise good judgement and airmanship; \\
\hline Meteorology; & Apply aeronautical knowledge; and \\
\hline
\end{tabular}

\footnotetext{
${ }^{15}$ Annex 1 - The ICAO Annex about Personnel Licensing.
} 


\begin{tabular}{c|c}
\hline Subjects of knowledge & Practical skills \\
\hline Navigation; & $\begin{array}{c}\text { Maintain control of the RPA at all times in a manner } \\
\text { such that the successful outcome of a procedure or } \\
\text { maneuver is assured. }\end{array}$ \\
\hline Operational procedures; & \\
\hline Principles of flight; and & \\
\hline Radiotelephony. & \\
\hline
\end{tabular}

Taking into consideration that the RIC is not onboard but in a different location from the UAS and especially, in BVLOS operations pilot and aircraft can be separated by hundreds or thousands of miles, it is critical that humanmachine interface (HMI) can provide the RIC with all the necessary data to perform the required tasks timely and accurately. UAS incorporate high level of automation oriented towards making the operations easier to be conducted and more efficient. However, the fact that automation may not always perform as intended must be considered. The work mentioned in Ref. [17], highlights that HMI must keep the UAS operator informed at all times about the automation's behavior and its intent. This work focuses on operating multiple UAS by a single operator but in terms of HMI design, it could be applied to single UAS operations.

Moreover, HMI must be at such a sophisticated level to prevent RIC from spending excessive time in performing basic piloting activities. Aviate, navigate, and communicate (not necessarily through voice) can be considered as basic piloting activities. So, HMI should facilitate the execution of these activities and also provide the means for performing effectively the main mission/operation for which the UAS took off in the first place. This could be achieved with controls that are easy to operate like sensors and other onboard devices. Furthermore, any data and information need to be easily accessible and readable. The level of HMI complexity increases linearly with the type of UAS. Smaller UAS are often controlled using remote controls in comparison to bigger UAS that are controlled by a Ground Control Station (GCS). In every case, the level of training shall be equivalent to the level of UAS complexity (GCS is included because it constitutes part of the UAS) and the complexity of the intended operations.

Although training is critical, there are no provisions for realistic RIC training. What is known in the manned aviation for its advantages is using flight simulators for the pilot training. While in bigger UAS may be provided with flight simulators like in the case of Predator ${ }^{16}$ training device, as shown in Ref. [32], for smaller UAS performing missions like inspections, fire-fighting, package delivery, etc., flight simulators are not provided, probably because they are considered as not cost-effective. Figure 6 shows the basic training that a RIC shall be provided with. (Ref. [18]).
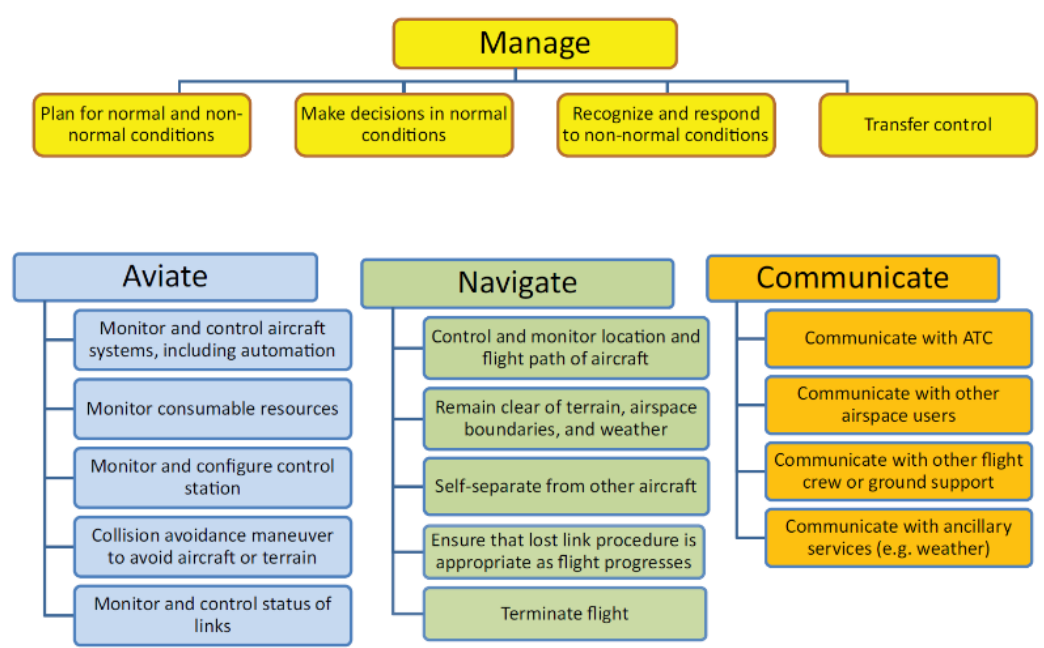

Fig. 6 A model of the responsibilities of a UAS pilot.

\footnotetext{
${ }^{16}$ Predator - General Atomics Aeronautical Systems Inc. (GA-ASI) MQ-9 Predator UAS.

'NASA's MQ-9 Ikhana Predator B had a wingspan of $66 \mathrm{ft}$ and is $36 \mathrm{ft}$ long, and a payload capability of greater than 400 pounds. Ikhana was powered by a Honeywell TPE 331-10T turbo-prop engine and could reach altitudes above 40,000 ft.' (Ref. [30]).
} 


\section{UAS Performance-based Concept}

As in manned aviation, PBO concepts are implemented in UAS operations too. Such concepts are applied almost in every kind of unmanned operation. UAS with the size, complexity, and cost of a manned aircraft, as well as, UAS that are smaller, relatively less complex, and may be used for recreational purposes should perform in a certain way in order to be eligible for operational approval. As already mentioned, UAS encompasses state of the art technology. One of the reasons could be the lack of pilot onboard, which necessitates UAS to perform almost perfect with minor deficiencies that in their majority would not affect flight safety. Application of PBO concepts is also a way to assure that the desired safety level will not be downgraded.

In line with PBO concepts, FAA formulate the basis of operational approval on the 'Performance Authorization' and 'Airspace Authorization' with the latter not being mandatory for operations in uncontrolled airspace. (Ref. [15]). The equipment requirements that are set by the FAA assures not only the desired level of performance but also the desired level of safety performance. So, it could be substantiated that PBO is a way of safety risk mitigation and the greater level of the required performance could mean a greater level of safety assurance.

\section{Safety Assurance and Operation Approval}

1) Safety Barriers

According to Ref. [19], the factors 'Regulations', 'Training', and 'Technology', are considered as factors that could drift an operation from its ideal performance (Baseline) to a more realistic one (Operational), see Fig. 7. Safety is maintained as long as performance is maintained between these states and the aforementioned factors work as Safety Barriers (SB). In the previous version of Ref. [19], these factors were considered as defenses that could impede accidents from happening, in an ideal situation. In real life, breaching all of these defenses or SB could lead to an accident. As already mentioned, ICAO suggests the mitigation strategies (MS) of Avoidance, Reduction, and Segregation.

In this paper, the distinct difference between SB and MS is that SB are mainly determined in an organizational level and applied well before the execution of an operation. On the other hand, MS are mainly applied shortly before the execution of an operation or even while the operation is being performed. For example, instant application of a Regulation is not possible. However, an instant directive not to violate a certain part of the airspace is possible and, in this case, it is a form of Segregation. Another way to translate MS is that when the risk of an activity needs to be diminished or degraded in terms of lowering the respective probability, then a possible solution is to invest in technology (e.g., use a better and more accurate system to perform an activity, or use TCAS in the case of MACs, etc.).

Considering the case of UAS, it is evident that technology for example, is widely used as a way of mitigation. The same applies for training and regulations. From the perspective of the oversight authorities and the authorities approving an operation, SB are used as mitigation barriers too. In addition, due to the fact that technology advances really quick, SB are also used as safety assurance measures. An indicative example is the FAA that requests certain performance level in order to issue Performance Authorization for UAS operations. Setting specific standards is not only a way of assuring a certain level of performance but also a way of mitigation.

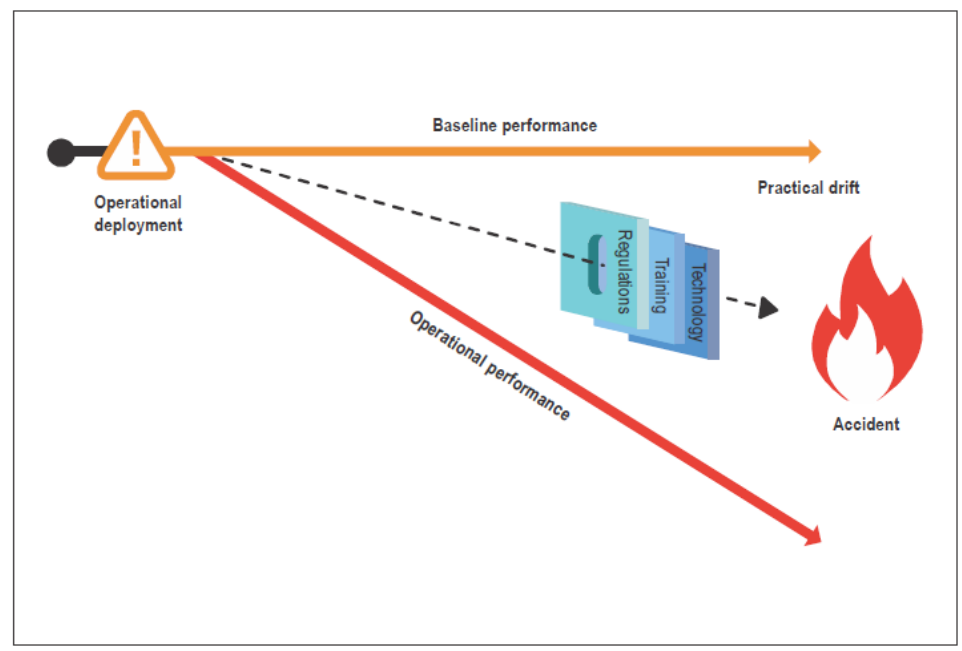

Fig. 7 The Practical drift Concept. 
Assessing the risk of an operator's point of view has the following characteristics, more or less similar to manned aviation. Considering a hypothetic scenario of an approved UAS operation, the following data could be assumed:

Firstly, the three SB are considered as factors that cannot be changed instantly (e.g., regulating instantly a new law). Also, the training of the RIC is already attained and the technology that is used has known advantages and disadvantages (e.g., a UAS with certain features). Secondly, based on the ICAO generic assessment, the risk of the operation is assessed as acceptable (green area). In a more detailed way, the operation valued as SAIL II (SORA process) or in the FAA context, the operation attained a 'Performance Authorization'. However, an accident did occur.

Similar to that scenario is the accident of a UAS that hit the ground after GPS-compass error. According to Ref. [2], the UAS after the error occurrence reverted from automated flight mode to manual flight mode. The pilot did not recognize the situation timely and the UAS went BVLOS, started descending until the time it hit the ground. The report mentions that the pilot was not required to be trained for emergency situations. In this accident the MS were in place (approval was granted). However, all the SB were breached. Technology did not work properly, Regulation for emergency training did not exist, and Training (the required one) did not exist too.

2) Main Similarities and Differences of CORUS and FAA ConOps

The main similarities are based on the fact that both ConOps use a performance-based concept for risk mitigation and allow UAS operation in both uncontrolled and controlled airspace. According to Ref. [29], an important similarity is that both ConOps use the mitigation layer concept of Strategic (pre-flight), Tactical, and Collision Avoidance in order to assure enough separation between aircraft. Moreover, data sharing (including flight data and operational intent) and cooperativeness are common between CORUS and FAA. Especially, the Strategic and Tactical deconfliction layers are based on data sharing. Also, cooperativeness is based on a form of data sharing because aircraft equipped with cooperative systems of separation need to exchange flight navigation data (e.g., altitude, airspeed, position, heading, rate of climb or descent) in order for such systems to work. Another important similarity is the RID that both ConOps consider as a way of significantly increasing the SA of UAS operators, manned aircraft pilots provided that they are able to receive that king of information, and as a means of performing oversight of every UAS operation conducted. Moreover, provisions are made for managing UAS traffic that may face emergency situations.

As far as the main differences are concerned, according to Ref. [29], in the EU a UAS operation is assessed for approval based on the UAS operation category (i.e., 'Open', 'Specific', and 'Certified') and the U-space services provided for that operation. In contrast, FAA does not have similar categorization and relies the operational approval on the performance standards that an operators shall meet. This paper considers the CORUS as more operator-oriented as it also provides UAS operators with the SORA process to assess and manage the safety risks linked to their operations. Conversely, the FAA ConOps is more authority-oriented based on the fact that the authority (FAA) not only provide the performance requirements but also holds the right to assess the overall risk level of an operation which may have met the required performance. Indeed, in the FAA ConOps there is no safety risk management tool like the SORA process. Moreover, the FAA describes proposals of how UAS may transmit RID and tracking information (i.e., 'Direct broadcast' and 'Network publishing'). FAA, also describes methods of increasing SA of manned and unmanned aircraft by the level of manned aircraft participation in the USS network (i.e., 'Passive' and 'Active' participation).

3) UAS Accidents

Apart from the aforementioned accident, in Ref. [1], a list of 59 UAS accidents from 2015 till 2019 are mentioned verifying the great involvement of technical failures and pilot errors. Another indicative UAS accident related to the technology used and the level of pilot training is reported from the Australian Transport Safety Bureau (ATSB). According to Ref. [4], a UAS collided with terrain after it had entered three aerodynamic stalls. From the first and the second stall there was sufficient altitude and the UAS self- recovered. Unlikely to the first and second, the third stall occurred in such an altitude that the remaining clearance with the terrain was insufficient resulting to the UAS collision. The pilots executed the emergency procedures promptly. However, the ATSB found that the consecutive aerodynamic stalls occurred due to the blockage in the pitot-static system that resulted in unreliable airspeed data being supplied to the autopilot. In the report, it is also mentioned that during the pre-flight checks, the pilots could have identified the erroneous airspeed indications. However, they did not.

From the aforementioned UAS occurrences the following considerations emerge:

- The redundancy of critical UAS systems could assist to avoiding accidents due to technical failures. Systems linked to the UAS navigation and flight characteristics were not built with a redundancy concept.

- The RIC training must be focused on emergency situations too. The exact description of every UAS system shall be documented and included in pilot training. 
- The UAS airworthiness remains the cornerstone of safety assurance. This ultimately linked to redundancy. It cannot be denied that in manned aviation, no aircraft during flight tests, entering consecutive aerodynamic stalls for any reason could achieve an airworthiness certification.

4) Critical Aspects

The three most critical factors affecting UAS safety assurance are the Level of Performance, Level of Cooperativeness, and the RIC Training. These factors are expressed through the levels of robustness and performance itself in the CORUS and FAA ConOps, respectively. Certification of the staff involved and the UAS based on specific standards aid in achieving these levels. The setting of proper and exact standards is also related to the level of autonomy of UAS. For example, today RIC are largely involved in controlling a UAS and perform a mission and for these reasons specific training is needed. The gap between current automated UAS and future autonomous UAS is technological.

Cooperativeness, in terms of various systems integration into UAS, drives the necessity for certification in order to assure the proper function of these systems and the fact that RIC will be able to operate these systems as well. Based on both ConOps, the greatest level of cooperativeness, the greatest the level of safety assurance will be. Additionally, an aspect of cooperativeness is the participation, or not, of UAS in the U-space network (CORUS) or USS network (FAA). Cooperativeness should be considered not only as the exchange of data between aircraft systems (e.g., TCAS) but also the sharing of flight information from one UAS operator to another. EASA and FAA apply stricter rules on non-cooperative traffic or even a form of segregation.

\section{Operational Scenarios}

The following scenarios investigate the transfer of people and goods delivery in VLL airspace and between $500 \mathrm{ft}$ AGL and 8,000 ft MSL. This upper limit has been put due to the fact that above 8,000 $\mathrm{ft}$ MSL pressurized passenger cabin is mandatory. Moreover, above that flight altitude most of the are conducted under IFR conditions which are out of the scope pf the paper based on Table 1. Pressurized cabins and IFR flight conditions direct different level of safety assurance and certification and so they are considered in the following OS.

Two types of routing are considered: 1. Over city (populated areas) and 2. Rural environment (non-populated areas). Missions like medical evacuation and first aid provision are not investigated. Generally, such missions are considered urgent so they should be subject to standing operational authorization. Both scenarios address the risks of MAC and GC in a BVLOS operating environment. Due to the fact that flights above 8,000 ft MSL must be performed by aircraft with cabin pressurization system, such UAS flights are not investigated. Further certification and safety assurance standards are needed for flights above that altitude.

In regard to SPTs and SPIs, they are ultimately linked to the safety risks of an operation. Based on [27], two BowTie ${ }^{17}$ diagrams are provided covering the case of MACs and GCs. In these examples, UAS are considered without people on board. In addition, the GC BowTie diagram has formed based on the assumption that UAS is flying at low altitude (i.e., below $500 \mathrm{ft}$ AGL). However, both examples could be used to form future SPTs and SPIs.

\section{A. People transfer}

1) Assumptions

Currently, people transfer is not allowed in the 'Specific' category of operations. However, the scenario investigates the possibility of using UAS with weight below $150 \mathrm{~kg}$ to transfer people. The minimum number of people is one. This means that in the future this type of mission could be similar to private transportation using cars. The desired achievable probability of a MAC and GC is zero and extremely improbable, respectively. Moreover, the SORA process and the FAA ConOps are not structured to cover the transfer of people but they will be considered as a basis.

2) Safety Assurance

Regarding ground risk, based on the SORA methodology, the options of BVLOS in sparsely populated environment and BVLOS in populated environment are considered. Table 14 depicts the respective values of ground risk that needs mitigation. SORA proposes three mitigation measures (i.e., Strategic mitigation, Effects of ground impact are reduced, and an emergency response plan (ERP) is in place, the UAS operator is validated and effective).

\footnotetext{
${ }^{17}$ This method is described through the free BowTieXP software. (www.cgerisk.com). It provides a visualization of the risk assessment process. In the middle, the main hazardous event is depicted, which is linked with the hazard (at the top of the diagram) based on type of operation. On the left-hand side there are the threats that release/enable the main hazardous event to occur and on the right-hand side there are the possible consequences. Between the main hazard and the two sides the barriers and mitigation measures are depicted.
} 
All the three should be applied in order to effectively minimize the ground risk. Moreover, in the case of populated areas, a ground risk class has not been developed yet. Taking into consideration that the level of robustness should be high and based on the JARUS description of the three mitigation measures, this scenario assumes that the most critical factors to determine the ground risk and then minimize it are the determination of people at risk on the ground and the validation from a third party of the UAS performance.

As far as the air risk is concerned, based on the SORA methodology, in this scenario, the most critical OSOs are considered to be the OSOs \#02, 05, 06, 10, 18, and 19. Similarly to the ground risk, the desired level of robustness should be high. Table 15 provides an indicative example concerning the determination of the robustness level for the OSO \#18. According to the SORA process both the level of integrity and level of assurance must be high in order to achieve an also high level of robustness. OSO \#18 refers to UAS operations where no people are on board. However, in UAS operations of people transferring the Automatic protection of the flight envelope from human errors considered to be a necessity.

Table 14 Determination of the Intrinsic UAS Ground Risk Class.

\begin{tabular}{|c|c|c|c|c|}
\hline \multicolumn{5}{|c|}{ Intrinsic UAS ground risk class } \\
\hline Max UAS characteristics dimension & $\begin{array}{l}1 \mathrm{~m} / \text { approx. } \\
3 \mathrm{ft}\end{array}$ & $\begin{array}{l}3 \mathrm{~m} / \text { approx. } \\
10 \mathrm{ft}\end{array}$ & $\begin{array}{l}8 \mathrm{~m} / \text { approx. } \\
25 \mathrm{ft}\end{array}$ & $\begin{array}{l}>8 \mathrm{~m} / \text { approx. } \\
25 \mathrm{ft}\end{array}$ \\
\hline Typical kinetic energy expected & $\begin{array}{l}<700 \mathrm{~J} \\
\text { (approx. } \\
529 \mathrm{ft} \mathrm{lb} \text { ) }\end{array}$ & $\begin{array}{l}<34 \mathrm{~kJ} \\
\text { (approx. } \\
25000 \mathrm{ft} \mathrm{lb)}\end{array}$ & $\begin{array}{l}<1084 \mathrm{~kJ} \\
\text { (approx. } \\
800000 \mathrm{ft} \mathrm{lb)}\end{array}$ & $\begin{array}{l}>1084 \mathrm{~kJ} \\
\text { (approx. } \\
800000 \mathrm{ft} \mathrm{lb} \text { ) }\end{array}$ \\
\hline \multicolumn{5}{|l|}{ Operational scenarios } \\
\hline $\begin{array}{l}\text { VLOS/BVLOS over a controlled } \\
\text { ground area }{ }^{3}\end{array}$ & 1 & 2 & 3 & 4 \\
\hline $\begin{array}{l}\text { VLOS in a sparsely populated } \\
\text { environment }\end{array}$ & 2 & 3 & 4 & 5 \\
\hline $\begin{array}{l}\text { BVLOS in a sparsely populated } \\
\text { environment }\end{array}$ & 3 & 4 & 5 & 6 \\
\hline VLOS in a populated environment & 4 & 5 & 6 & 8 \\
\hline BVLOS in a populated environment & $\mathrm{TBD}^{4}$ & $\mathrm{TBD}^{4}$ & $\mathrm{TBD}^{4}$ & $\mathrm{TBD}^{4}$ \\
\hline VLOS over an assembly of people & 7 & & & \\
\hline BVLOS over an assembly of people & $\mathrm{TBD}^{4}$ & & & \\
\hline
\end{tabular}

Table 15 OSO \#18 Robustness determination.

\begin{tabular}{|c|c|c|c|}
\hline \multirow{2}{*}{\multicolumn{2}{|c|}{ HUMAN ERROR }} & \multicolumn{2}{|c|}{ LEVEL of INTEGRITY } \\
\hline & & Low & Medium \\
\hline \multirow{2}{*}{$\begin{array}{l}\text { OSO \#18 } \\
\text { Automatic } \\
\text { protection of } \\
\text { the flight } \\
\text { envelope from } \\
\text { human errors }\end{array}$} & Criteria & $\begin{array}{l}\text { The UAS flight control system incorporates automatic } \\
\text { protection of the flight envelope to prevent the remote } \\
\text { pilot from making any single input under normal operating } \\
\text { conditions that would cause the UA to exceed its flight } \\
\text { envelope or prevent it from recovering in a timely fashion. }\end{array}$ & $\begin{array}{l}\text { The UAS flight control system incorporates automatic protection of the } \\
\text { flight envelope to ensure the UA remains within the flight envelope or } \\
\text { ensures a timely recovery to the designed operational flight envelope } \\
\text { following remote pilot error(s). }\end{array}$ \\
\hline & Comments & N/A & $\begin{array}{l}{ }^{1} \text { The distinction between a medium and a high level of robustness for } \\
\text { this criterion is achieved through the level of assurance (see table } \\
\text { below). }\end{array}$ \\
\hline
\end{tabular}

\begin{tabular}{|l|l|l|l|l|}
\hline \multicolumn{2}{|c|}{ HUMAN ERROR } & \multicolumn{1}{c|}{ LEVEL of ASSURANCE } \\
\cline { 3 - 5 } & & \multicolumn{1}{c|}{ Low } & \multicolumn{1}{c|}{ Medium } \\
\hline \begin{tabular}{l} 
OSO \#18 $\begin{array}{l}\text { Automatic } \\
\text { protection of } \\
\text { the flight } \\
\text { envelope from } \\
\text { human errors }\end{array}$ \\
\cline { 3 - 5 }
\end{tabular} & Criteria & $\begin{array}{l}\text { The automatic protection of the flight } \\
\text { envelope has been developed in-house or out } \\
\text { of the box (e.g. using commercial off-the-shelf } \\
\text { elements), without following specific } \\
\text { standards. }\end{array}$ & $\begin{array}{l}\text { The automatic protection of the flight envelope } \\
\text { has been developed to standards considered } \\
\text { adequate by the competent authority and/or in } \\
\text { accordance with a means of compliance } \\
\text { acceptable to that authority. }\end{array}$ & $\begin{array}{l}\text { Same as Medium. In addition, } \\
\text { evidence is validated by EASA. }\end{array}$ \\
N/A & N/A & N/A \\
\hline
\end{tabular}

Based on the fact that operations of this scenario will be conducted without pilot on board (only people to be transferred), the OSO \#18 is mandatory. In addition, a possible RIC may not be able to intervene and always control the UAS due to link continuity issues. Moreover, in the case of single human transferring, it is unlikely that everyone may be a pilot or have piloting background. These considerations lead to the conclusion that the level of autonomy needs to be raised. Another important aspect is that designing standards for UAS performing such operations are not 
defined yet, at least in a worldwide basis. These standards will define the expected target level of safety and thus the required SPIs and SPTs.

In regard to C3 link, similarly to the OSO \#06 (C3 link characteristics), an OSO needs to be developed to cover the event of C3 lost procedures. Factors affecting the performance of a link are the transaction expiration time, the availability, the continuity, and the integrity. (Ref. [8]). Altitude is a parameter that seriously affects the link performance, especially its continuity. Indeed, in urban flight environment the case of loss of Line of Sight (LOS) is considerable due to the existence of buildings that differentiate largely on their height. Loss of LOS may cause link interruption between RIC (or ground station) and the UAS itself causing loss of UAS control. On the other hand, UAS operations in higher altitude and in rural environment may suffer less from the loss of LOS.

So, based on different challenges, different SPIs needs to be developed in order to monitor and measure in real time the link performance. In cases where link interruption is possible and may deteriorate the UAS operation, the use of a Link Relay Aircraft (LRA) should be considered. LRA could be used instead of satellites as a less expensive and more controllable option to assist UAS operators in assuring the link performance and at the same time assist competent authorities (e.g., CAAs) in monitoring the link performance of the UAS operating in a certain area and issue any required advisory.

About MACs, the scenario considers the cooperativeness between different traffic to be essential for achieving timely and adequate separation. The safety layers described in both CORUS and FAA ConOps may be adequate. However, they rely on the fact that information and data relevant to each flight are disseminated and shared to other UAS operators as well. In the event of conflicting with a non-cooperative traffic, the safety assurance cannot be achieved, at least at the desired level for this scenario. For non-cooperative traffic, the remote ID and the sharing of certain flight data and operation intent with the USS or USSP may provide a level of deconfliction. The question is how reliable these data are and how fast they can be processed and provided to the relevant UAS operators.

Finally, the safety of the people on board needs to be examined from the aspect of ground procedures. For example, where would UAS transferring people operate from? How people may be assisted to enter the UAS, sit and tight their seatbelts? Similar are the considerations for the disembarkation of people.

\section{3) Certification}

For the certification of UAS performing people transferring relevant standards should be developed that do not exist at the time of writing. Referring to UAS under $150 \mathrm{~kg}$, weight is probably the most critical factor related to certification. The reason for this is that such UAS needs to be certified accordingly in order to achieve the desired level of safety which in this scenario is similar to that of manned aviation people transferring. That level of safety requests certain systems to be installed on the aircraft for traffic and ground collision avoidance. The algorithm that controls and directs the evasive maneuver in an imminent impact should be considered in terms of the operating environment (i.e., city or rural). Over cities and in VLL there is less airspace available to execute a maneuver than in rural areas and above $500 \mathrm{ft}$ AGL. For UAS operating in, but not limited to, VLL, algorithms of traffic collision avoidance need to take into account the existence of slow-moving aircraft and helicopters.

Concerning the certification of RIC, as long as the required standards have been developed and the relevant training is applied tailored to the specificities of each UAS, the desired level of safety could be attained.

From an ATC perspective, safety assurance is achieved as long as oversight and performance monitoring systems are in place. Due to the huge number of future UAS served as air-taxis and the already big amount of UAS operators, the development of such systems would assist in maintain safety. Oversight and Performance monitoring regions (OPRs), like Flight Information Region (FIR) in manned aviation, should be developed. Parts of the U-space that are not certified as OPR should not permit the execution of complex operations with increased level of safety target.

4) Developing SPTs and SPIs for OS-A

Based on the 'Threats' depicted in Fig. 8 and Fig. 9, SPTs can be developed for UAS operation. Additional 'Threats' could be added or new could be developed based on each operation specificities. Similarly, the respective SPIs are formed. For example, for the Threat related to on board systems failures (last threat of Fig. 9), a SPT could be to decrease the number of failures of a certain UAS systems (e.g., GPS, flight control system, C3 link, etc.). Next, possible SPIs could be the frequency of a certain system failure (e.g., number of GPS failures or degraded performance during a 100 flight-hour period.

Taking as a reference the OSOs mentioned in the SORA methodology, a UAS operator shall develop the respective set of SPT and SPI. This will enhance the operation performance monitoring and thus will add to its safety assurance.

The level of UAS autonomy may drive the development of different set of SPT and SPI or update the existing. Indeed, the level of technology and automation may result to a decreased frequency of UAS systems failures. Moreover, high level automation may provide the UAS operators with the ability to perform effective oversight of 
their UAS operations, monitoring UAS performance, and intervene when it is deemed necessary. In order to streamline the performance of UAS, SPIs need to be monitored during the execution of a flight. 


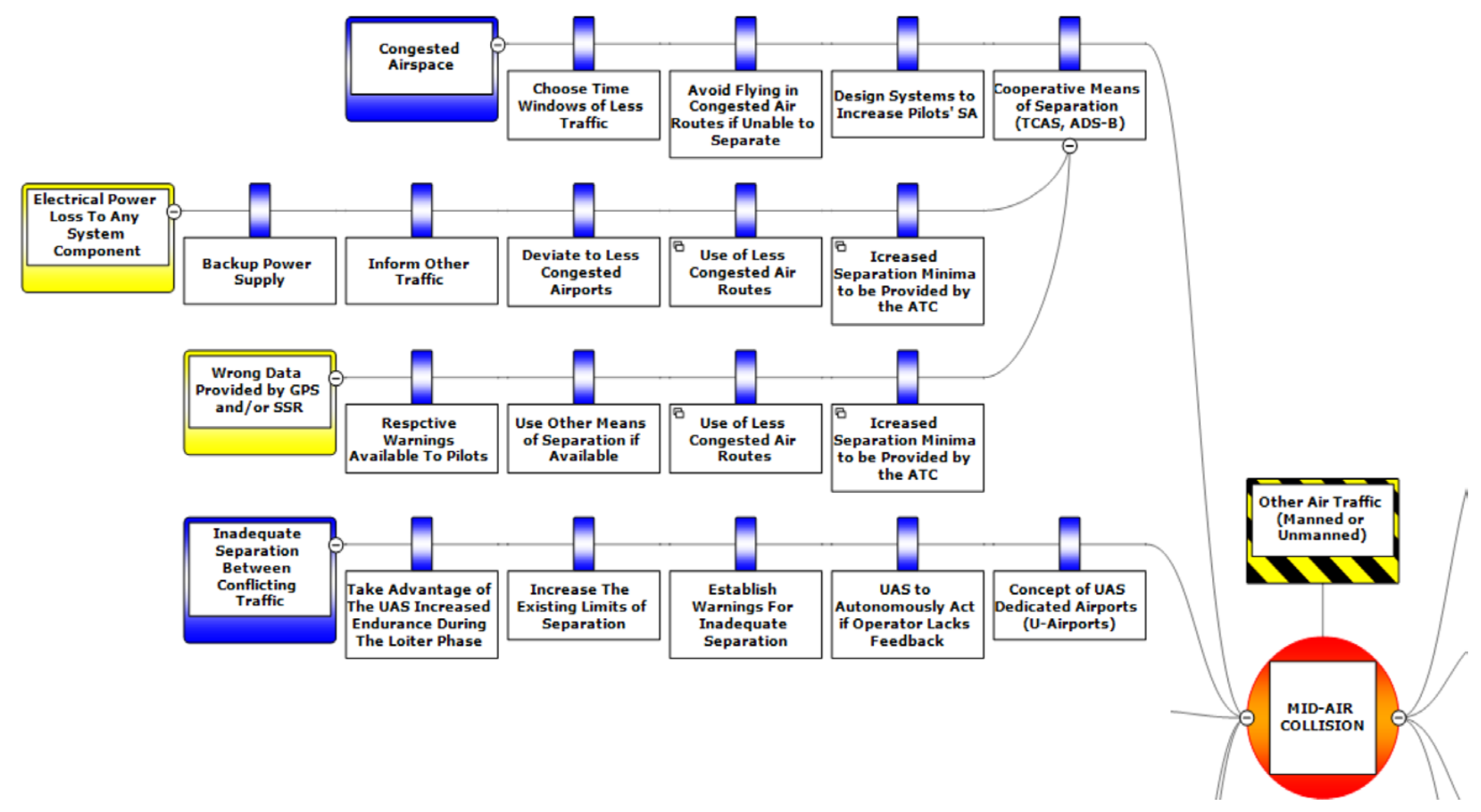

Fig. 8 MAC threats (a). 


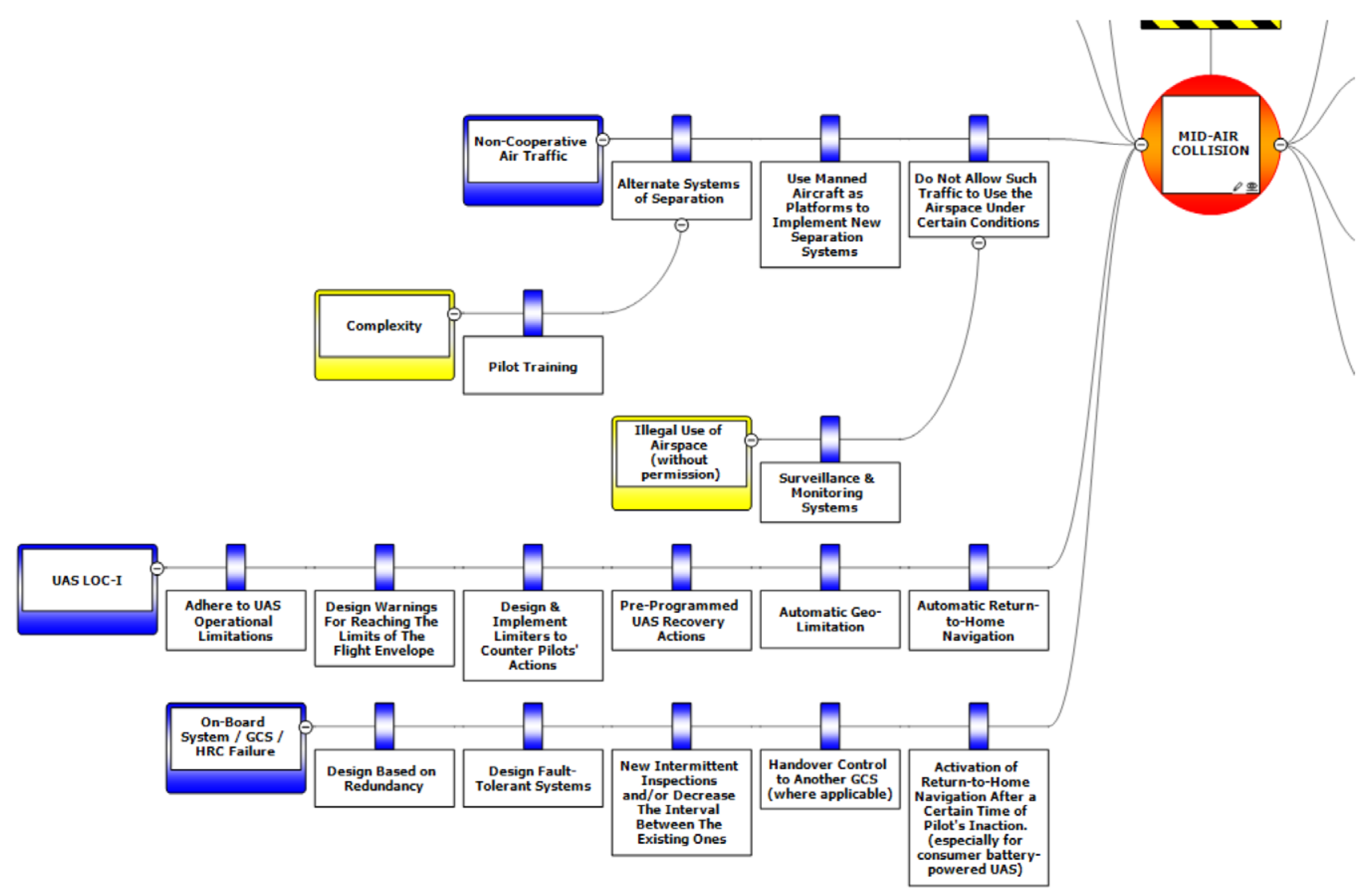

Fig. 9 MAC threats (b). 


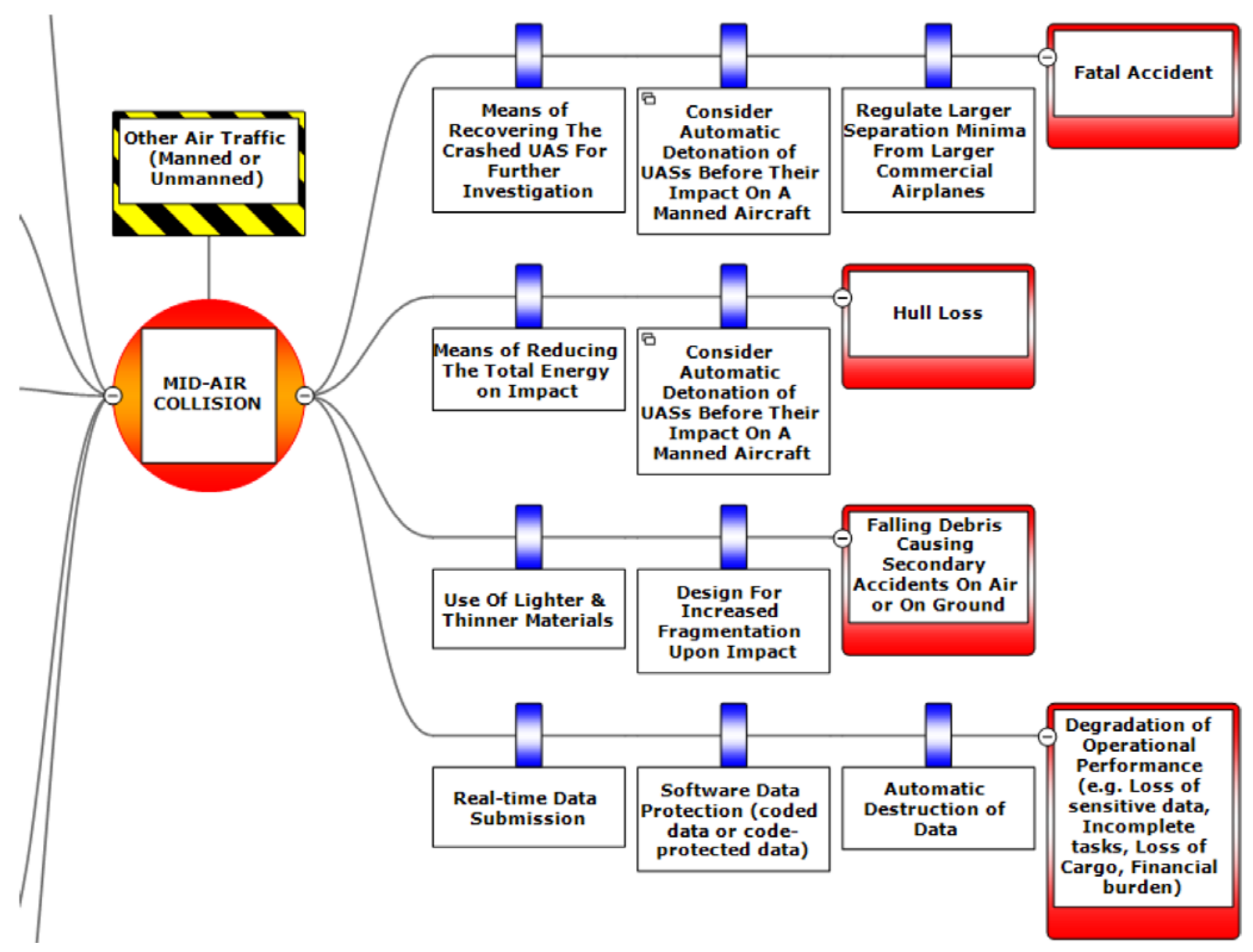

Fig. 10 MAC consequences. 


\section{B. Goods delivery}

1) Assumptions

In the Easy Access Rules for UAS (2020), the carriage of dangerous goods is one of the UAS operations contained in the 'Certified' category. In parallel, the 'Specific' category does not exclude the transferring of goods. This scenario assumes that UAS carry a maximum weight that corresponds to $25 \%$ of its Maximum Takeoff Weight (MTOW). Generally, the scenario deals with small package delivery, mail delivery, and single purchased items. Additionally, the carried items are contained in the UAS or in the case they are hanged underneath the UAS main part, items do not impede the aerodynamic stability and thus the entire flight performance of the UAS is not affected. The desired achievable probability of a MAC and GC is zero and improbable, respectively. Basically, the SORA process is used.

\section{2) Safety Assurance}

Regarding ground risk, the same considerations with OS-A concerning the level of population apply to this scenario too. Moreover, OS-B could materialize in routings where no population exist. In that case the values of Table 14 need to be updated and particularly the respective values for no populated routings and even less populated areas could be lower than those depicted. The aforementioned mitigation measures can be applied. Selection of the landing site is a factor affecting safety assurance and certification. In case of a remote landing site, safety can be easily assured using standards similar to manned aviation landing sites. However, UAS conducting such kind of delivery are expected to deliver products in the same location to the location of the buyer (e.g., a house or a property). The concern that arises is that OS-B operations should be conducted only if the buyer's landing site is available and certified. Currently, such standards have not been developed. Moreover, the procedural part of offloading a package must be fully automated due to the fact that it is not possible to train every human how to handle a particular UAS.

As far as the air risk, the risk of MACs, and the risk C3 link loss are concerned, the same considerations of OS-A are applicable to OS-B too.

\section{3) Certification}

For this scenario, the certification considerations of OS-A are also applicable. In addition, specific certification is needed in line with the different types of package drop-off. UAS may either land and the package received by the buyer or the UAS may hover and release the package from a certain altitude using a parachute or similar technique to safely arrive on the ground. These certification aspects should be couple with the people (potential buyer) training to receive goods by using UAS. Moreover, wind characteristics is also an aspect that needs to be considered. In case packages are carried outside of the main airframe of a UAS, increased wind velocities may hinder the aerodynamic stability of UAS.

4) Developing SPTs and SPIs for OS-B

The considerations mentioned in 'Developing SPTs and SPIs for OS-A' are also applicable to OS-B. Moreover, in terms of 'Consequences', based on the aforementioned accidents and the assumption that in case of a GC the total energy will be fatal to people who may get hit, the respective risk probability must be zeroized. The severity of a GC cannot be realistically minimized to zero, so the only way of mitigation is to avoid the accident itself. Like manned aircraft, UAS may collide to the ground for several reasons. At least for those reasons connected to UAS systems or parts of them, some of them being crucial for UAS performance as a flying machine, redundancy must be assured. The CS based on which UAS are manufactured shall be part of the safety risk management and assurance process. 


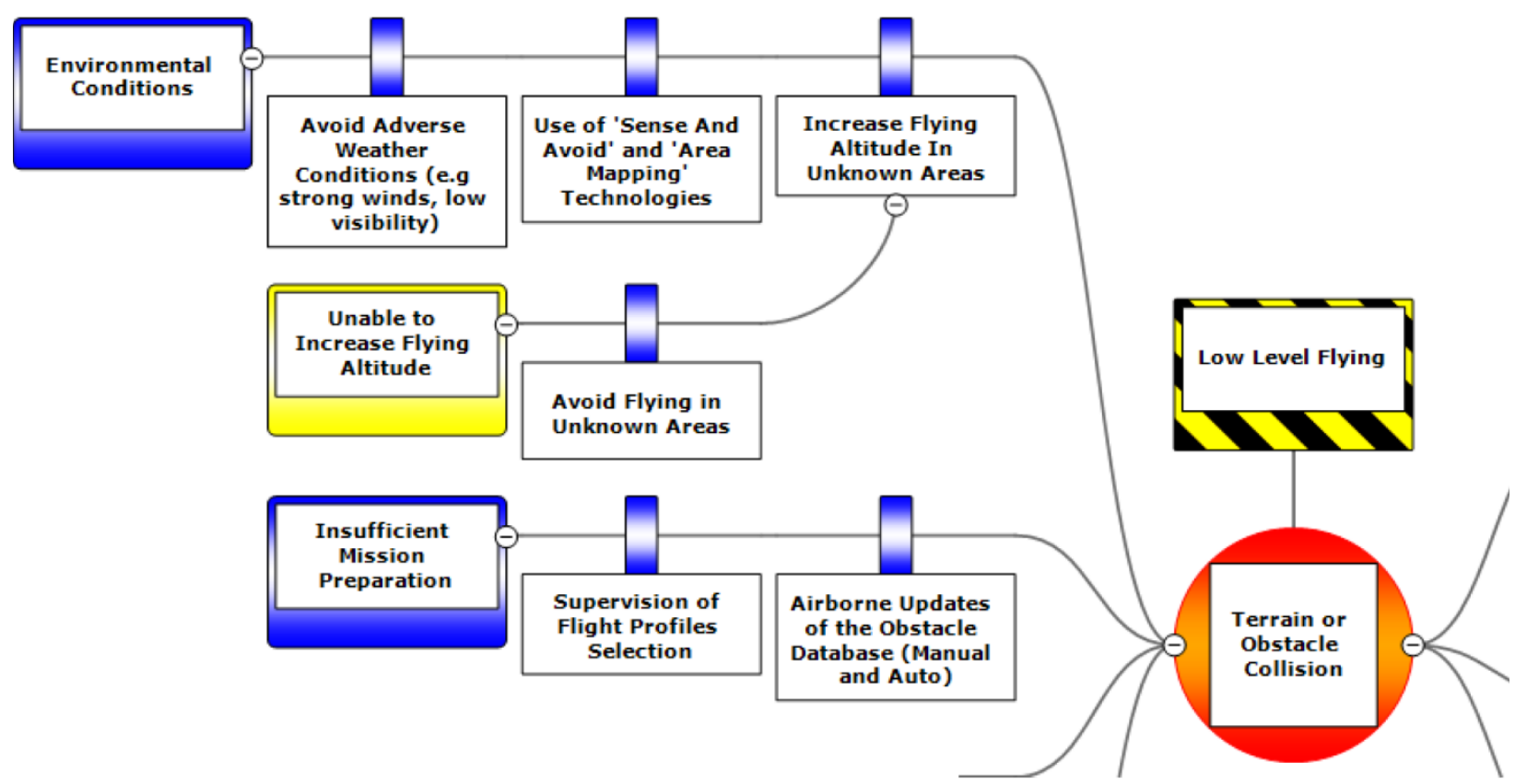

Fig. 11 GC threats (a).

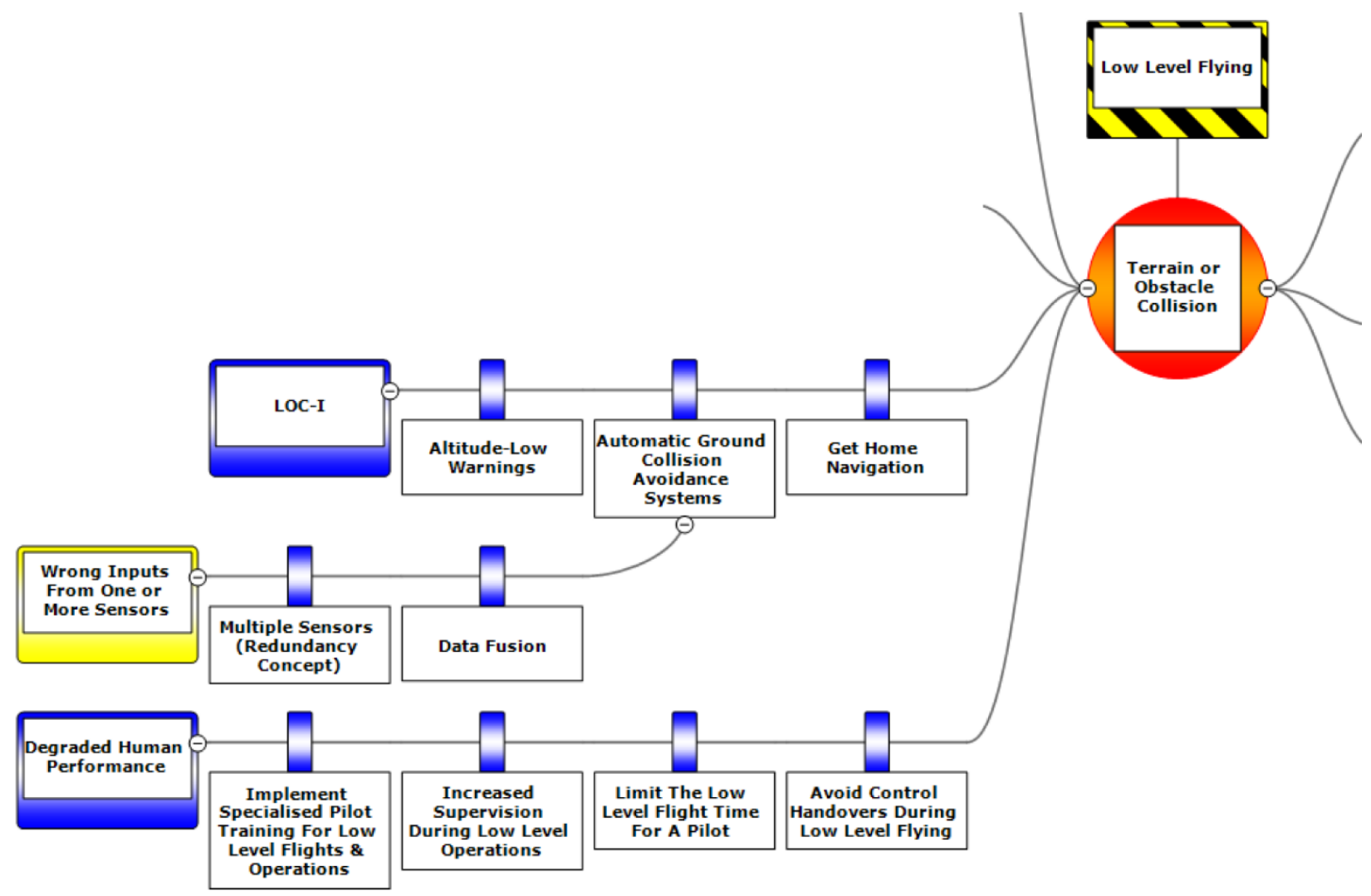

Fig. 12 GC threats (b). 


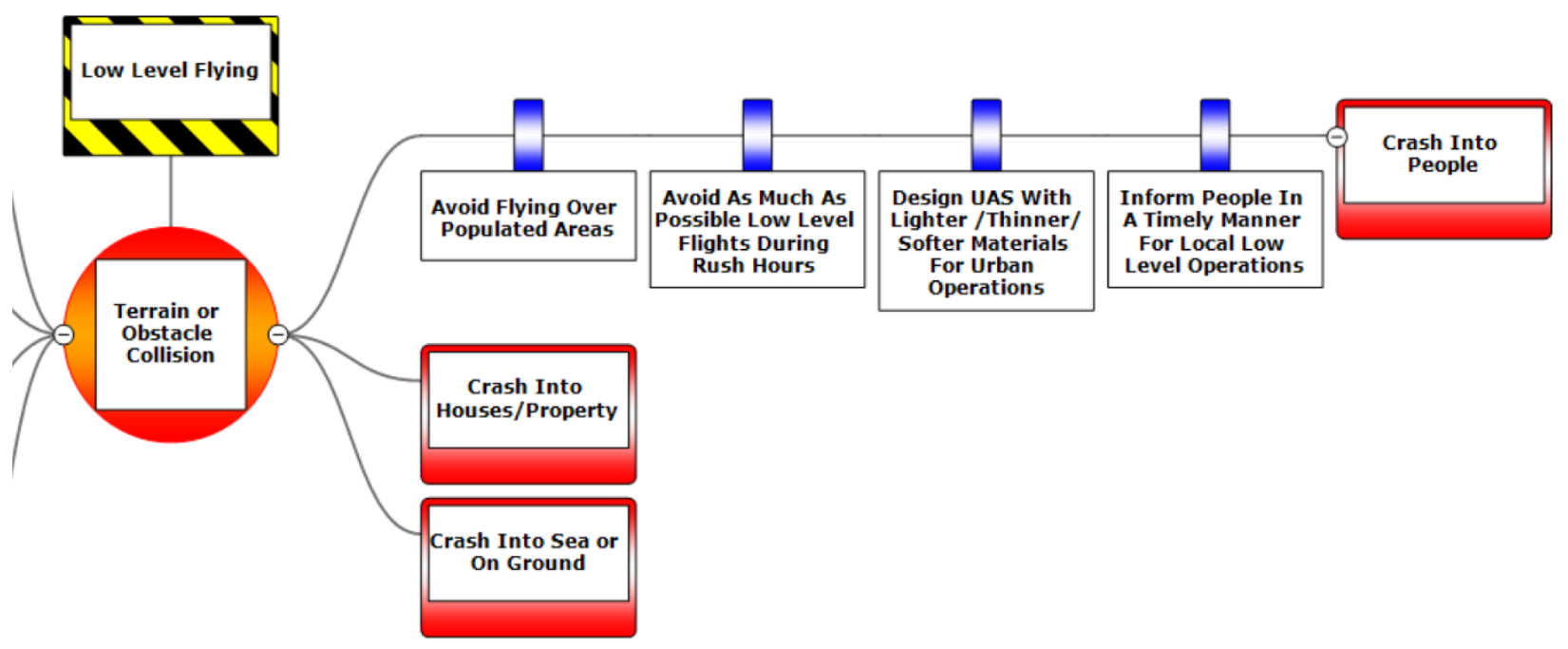

Fig. 13 GC consequences.

\section{Findings and Recommendations}

\section{A. Findings}

From the comparison of safety assurance and certification in manned and unmanned aviation and the two OS of future UAS operations the following findings have been identified:

1) Strategic, Tactical, and Collision avoidance techniques shall be used to mitigate safety risks in any UAS operation.

2) Current technology provides techniques of ensuring separation mostly between cooperative traffic.

3) Safety assurance in areas where cooperative and non-cooperative aircraft operate cannot be guaranteed, especially during off nominal situations.

4) Data sharing and flight information exchange are the basic components of safety assurance and oversight.

5) SA and thus effective UAS control cannot be maintained without data sharing.

6) Off nominal situations mandate the need of data sharing.

7) Provisions and certification for loss of C3 link procedures shall be developed.

8) Safety assurance and certification capabilities are linearly linked to the existing UTM levels and generally to the existing technology and UAS performance.

9) Certification Standards need to be developed for the upcoming UAS operations both for the design of such UAS and for the operation execution.

10) The lack of safety metrics like SPTs and SPIs in UAS operations hinders effective oversight of safety performance and renders safety assurance less objective.

11) System redundancy is of paramount importance for maintaining and advancing safety assurance levels. As technology progresses redundancy concepts of designing UAS should be widely applied.

12) Unlike to performance, the level of systems redundancy has not been considered in any UAS ConOps so far.

13) ATC services cannot maintain sufficient SA for every manned and unmanned traffic under their control. High levels of SA are only available when the relevant technology is in place and work properly. In case of degraded operating systems either from the UAS or the ATC side, the provision of flight information and other ATC directions are significantly questionable.

14) The SORA methodology is mostly based on the procedural aspect of assuring UAS operations safety. The aspect of performance assurance as a means of safety assurance is clearly described by the FAA ConOps. 


\section{B. Recommendations}

Based on the findings already mentioned, the following recommendations are made:

1) Further division of the 'Specific' category of UAS operations is needed.

2) This division could enhance the development of specific SPIs and SPTs for each type of operation.

3) Mitigation for the risk of C3 link loss shall be developed. Currently, this safety issue could be addressed by using LRA instead of using satellites.

4) Develop technology and procedures in order to achieve effective oversight and information provision for every UAS flying in a certain part of the airspace.

5) The level of automation shall be taken into account during safety risk assessment.

6) The term 'cooperative' shall expand to cover the fact of a UAS participating or not in a network and exchanges flight data with other UAS and/or manned aircraft. Like 'cooperative' and 'non-cooperative', the term 'participating' or 'non-participating' UAS or manned aircraft shall be established. 'Participating' UAS may receive easier operational approval in contrast to 'non-participating' UAS. Also, the level of 'participation' needs to be taken into account in the safety risk management and assurance process.

7) In line with the SORA process, more PDRAs needs to be developed that take into account the new technological improvements and thus ease the process of operational approval for UAS operations that are conducted in a regular basis.

8) The concept of Network participating and the respective level of participation for data exchange, as described in the FAA ConOps, should be part of the safety risk assessment and operational approval process. Generally, as the level of Network participation increases the operation safety risk level should decrease.

\section{Conclusion}

In conclusion, creating a SMS(U) is not expected to cover the entire unmanned aviation activities and every UAS type. However, it should cover the activities and the corresponding spectrum of UAS types that are intended to perform unmanned operations while effectively assuring the management of safety risks that are derived from the simultaneous operation of manned and unmanned aircraft. The fundamentals that can be easily transferred from the manned aviation SMS, regarding Safety Assurance and Certification aspects, need to be studied from operation-centric perspective and developed as to be optimized for each specific operation. This operation-centric approach leads to a proposed further division of the 'Specific' category based on indicative contemporary UAS operations in order to facilitate the optimization of Safety Assurance and Certification taking into account the complexity of each operation. In all the ConOps mentioned before, their common characteristic is the collaboration between ATM and UTM as well as the provision of aeronautical information to UAS operators. Increased collaboration, effective oversight of UAS operations, and introduction of redundancy-based UAS design concepts will significantly add to the ongoing endeavour of UAS full integration to current airspace.

\section{Acknowledgments}

The author thanks Dr. Panagiotakopoulos, Dr. Braithwaite, and Dr. Tsourdos for their invaluable help towards finding data relevant to the completion of this paper.

[1]. Air Accidents Investigation Branch (AAIB) Bulletin, 1/2020. assets.publishing.service.gov.uk. [Online] URL: https://assets.publishing.service.gov.uk/government/uploads/system/uploads/attachment_data/file/919996/AAIB_Bulletin_12020_Hi_Res.pdf [retrieved 1 June 2021].

[2]. Air Accidents Investigation Branch (AAIB) Bulletin, 7/2020. assets.publishing.service.gov.uk. [Online] URL: https://assets.publishing.service.gov.uk/government/uploads/system/uploads/attachment_data/file/920095/AAIB_Bulletin_72020_Hi_Res.pdf

[retrieved 1 June 2021]. 
[3]. AIRBUS S.A.S, "CityAirbus Our four-seat eVTOL demonstrator", 2021. www.airbus.com. [Online] URL: [retrieved 12 May 2021].

[4]. Australian Transport Safety Bureau (ATSB), "Collision with terrain involving an Insitu ScanEagle X200 unmanned aircraft $\begin{array}{llll}\text { system } & \text { (UAS)", } & \text { www.atsb.gov.au. }\end{array}$ URL: $\quad$ https://www.atsb.gov.au/media/5776567/ao-2019-004_final.pdf [retrieved 2 June 2021].

[5]. Coventry City Council, "World-first electric Urban Air Port® secures UK government backing", 2021. www.coventry.gov.uk. [Online]

URL: https://www.coventry.gov.uk/news/article/3691/world-first_electric_urban_air_port_secures_uk_government_backing [retrieved 12 May 2021].

[6]. DJI, "MAVIC 2 Pro/Zoom User Manual V2.2", 2018. dl.djicdn.com. [Online] URL: $\quad$ https://dl.djicdn.com/downloads/Mavic_2/Mavic_2_Pro_Zoom_User_Manual_v2.2_en.pdf [retrieved 14 May 2021].

[7]. Downs, E. C., 2011. Jane's Avionics 2011-2012. 13th ed. Coulsdon: IHS Jane's.

[8].EASA, "Easy Access Rules for Unmanned Aircraft Systems", 2021. www.easa.europa.eu. [Online] URL: https://www.easa.europa.eu/sites/default/files/dfu/Easy\%20Access\%20Rules\%20for\%20Unmanned\%20Aircraft\%20Systems $\underline{0 . p d f}$ [retrieved 1 June 2021].

[9]. EASA, "Guidelines on Design verification of UAS operated in the 'specific' category and classified in SAIL III and IV", 2021. www.easa.europa.eu. URL:
[retrieved 25 May 2021]

$\begin{array}{lllll}\text { [10]. EASA, "Part-Flight } \quad \text { Crew Licensing } & \text { (FCL)", } & \text { 2016. ww.easa.europa.eu. }\end{array}$ URL: $\quad$ https://www.easa.europa.eu/sites/default/files/dfu/Part-FCL.pdf [retrieved 30 May 2021].

[11]. EASA, "Policy statement: Airworthiness certification of Unmanned Aircraft Systems" , 2009. www.easa.europa.eu. [Online] URL: $\quad$ https://www.easa.europa.eu/sites/default/files/dfu/E.Y013-01_\%20UAS_\%20Policy.pdf [retrieved 25 May 2021].

[12]. European Union, "COMMISSION IMPLEMENTING REGULATION (EU) 2018/1048", 2018. Official Journal of the European Union, eur-lex.europa.eu. [Online] URL: $\quad$ https://eur-lex.europa.eu/legal-content/EN/TXT/PDF/?uri=CELEX:32018R1048\&from=EN [retrieved 20 May 2021].

[13]. FAA, Department Of Transportation "Operation of Small Unmanned Aircraft Systems Over People", 2021. www.faa.gov. [Online]

URL:

[retrieved 2 June 2021].

https://www.faa.gov/news/media/attachments/OOP_Final\%20Rule.pdf

[14]. Federal Aviation Administration (FAA), "Summary Of Small Unmanned Aircraft Rule (Part 107)", 2016. www.faa.gov. [Online]

URL:
[retrieved 25 May 2021].

https://www.faa.gov/uas/media/Part_107_Summary.pdf

[15]. Federal Aviation Administration (FAA), "UTM Concept of Operations Version 2.0", 2020. www.faa.gov. [Online] URL: $\quad$ https://www.faa.gov/uas/research_development/traffic_management/media/UTM_ConOps_v2.pdf [retrieved 14 May 2021].

[16]. Flight Safety Foundation (FSF), "Benefits Analysis of Space-Based ADS-B", 2016. flightsafety.org. [Online] URL: [retrieved 8 June 2021].

[17]. Friedrich, M., Vollrath, M., "Human-Machine Interface Design for Monitoring Safety Risks Associated with Operating Small Unmanned Aircraft Systems in Urban Areas", 2021. www.mdpi.com. [Online] URL: $\quad$ https://www.mdpi.com/2226-4310/8/3/71/htm [retrieved 5 June 2021].

[18]. Hobbs, A.; Lyall, B., "Human Factors Guidelines for Unmanned Aircraft Systems", 2016. journals.sagepub.com. [Online] URL: [retrieved 28 May 2021].

[19]. ICAO Doc 9859: "Safety Management Manual" Fourth Edition, 2018. SKYbrary. [Online] URL: $\quad$ https://www.skybrary.aero/bookshelf/books/5863.pdf [retrieved 12 May 2021].

[20]. ICAO, "Annex 6: Operation of Aircraft", 2016. Part I: International Commercial Air Transport - Aeroplanes. 10th ed. Montréal: ICAO.

[21]. ICAO, "Doc 4444: Procedures for Air Navigation Services - Air Traffic Management", 2016. Chapter 5: SEPARATION METHODS AND MINIMA. 16th ed. Montréal: ICAO. 
[22]. ICAO, "Doc 9997: Performance-based Navigation (PBN) Operational Approval Manual", 2015. 2nd ed. Montréal: ICAO.

[23]. ICAO, "Performance-based Navigation (PBN) Manual", 2013. www.peter2000.co.uk. [Online] URL: $\quad$ https://www.peter2000.co.uk/aviation/icao-documents/DOC-9613 PBN-MANUA R04 01JAN13.pdf [retrieved 20 May 2021].

[24]. ICAO, 2011. Unmanned Aircraft Systems (UAS) Cir 328-AN/190. www.icao.int. [Online] URL: $\quad$ https://www.icao.int/Meetings/UAS/Documents/Circular\%20328_en.pdf [retrieved 10 July 2018].

[25]. ICAO, 2015 . Doc 10019, Manual on Remotely Piloted Aircraft Systems (RPAS) First Edition. servicos.decea.gov. [Online] URL: [retrieved 10 July 2018].

[26]. Jane's by IHS Markit, 2017. Jane's Flight Avionics 2017-2018. s.l.:David Cleland-Smith.

[27]. JARUS, "JARUS guidelines on $\quad$ SORA", $2019 . \quad$ jarus-rpas.org. [Online] URL: $\quad$ http://jarus-rpas.org/sites/jarus-rpas.org/files/jar_doc_06_jarus_sora_v2.0.pdf [retrieved 16 May 2021].

[28]. Karyotakis, M. K., 2019. "Safety Risk Management". In: MSc Project: Unmanned Aircraft Systems (UASs) in the 21st century. On attaining safer UAS flights based on current and future challenges and considerations.. London: City University of London, pp. 76-84.

[29]. Lieb, J., Volkert, A., "Unmanned Aircraft Systems Traffic Management: A comparison on the FAA UTM and the European CORUS ConOps based on U-space", 2020. www.researchgate.net. [Online] URL: https://www.researchgate.net/publication/345762481_Unmanned_Aircraft_Systems_Traffic_Management_A_comparison_o n_the_FAA_UTM_and_the_European_CORUS_ConOps_based_on_U-space [retrieved 5 June 2021].

[30]. NASA, "NASA Armstrong Fact Sheet: Ikhana Predator B Unmanned Science and Research Aircraft System", 2019. www.nasa.gov.

URL: [retrieved 30 May 2021].

[Online]

1]. National Aeronautics and Space Administration (NASA), "UAM Market Study", 2018. NASA Technical Reports Server. [Online]

URL: [retrieved 12 May 2021].

https://ntrs.nasa.gov/citations/20190001472

[32]. Rowe, L. J., Conwell, S. L., Morris, S. A. \& Schill, N. P., 2015. Volume 5 - Section XVIII: Human Factors and Training Chapter 104: Using Best Practices as a Way Forward for Remotely Piloted Aircraft Operators: Integrated Combat Operations Training-Research Testbed. In: Handbook of Unmanned Aerial Vehicles. New York London: SpringerReference, pp. 25052523.

[33]. Royal Aeronautical Society, 2021. "Call the eVTOL air ambulance". AEROSPACE Journal, January, Vol. 48, No. 1, p. 35.

[34]. Royal Aeronautical Society, 2021. "Exclusive: Y6S Plus unveiled". AEROSPACE Journal, January, Vol. 48, No. 1, p. 5.

[35]. SESAR JOINT UNDERTAKING, Concept of operations for European Unmanned Traffic Management (UTM) systems (CORUS), "U-space Concept of Operations, Enhanced Overview", 2019. www.eurocontrol.int. [Online] URL: $\quad$ https://www.eurocontrol.int/project/concept-operations-european-utm-systems [retrieved 14 May 2021].

[36]. SESAR JOINT UNDERTAKING, Concept of operations for European Unmanned Traffic Management (UTM) systems (CORUS), "U-space Concept of Operations", 2019. www.eurocontrol.int. [Online] URL: $\quad$ https://www.eurocontrol.int/project/concept-operations-european-utm-systems [retrieved 14 May 2021].

[37]. UK Civil Aviation Authority (CAA), "CAP 722: Unmanned Aircraft System Operations in UK Airspace - Guidance", 2020. publicapps.caa.co.uk. URL: [retrieved 15 May 2021].

[Online]

https://publicapps.caa.co.uk/docs/33/CAP722\%20Edition8(p).pdf

[38]. UK Civil Aviation Authority (CAA), "CAP 722A: Unmanned Aircraft System Operations in UK Airspace - Operating Safety Cases", 2019.

publicapps.caa.co.uk.

[Online] URL: https://publicapps.caa.co.uk/docs/33/CAP722A-UASOSC.pdf

[retrieved 20 May 2021]. 
2021-07-28

\section{Aspects and challenges of unmanned aircraft systems safety assurance and certification for advanced operations}

Karyotakis, Michail K.

AIAA

Karyotakis MK, Panagiotakopoulos D, Braithwaite G, Tsourdos A. (2021) Aspects and challenges of unmanned aircraft systems safety assurance and certification for advanced operations. In: AIAA Aviation 2021 Forum, 2-6 August 2021,Online https://doi.org/10.2514/6.2021-2397

Downloaded from Cranfield Library Services E-Repository 